Supporting Information

for

\title{
Ab initio and Density Functional Theory Evidence on the Rate- \\ Limiting Step in Morita-Baylis-Hillman Reaction
}

\author{
Dipankar Roy and Raghavan B. Sunoj * \\ Department of Chemistry \\ Indian Institute of Technology Bombay \\ Powai, Mumbai 400076, India
}

E-mail: sunoj@chem.iitb.ac.in

Fax: $91-22-2572-3480$ or $91-22-2576-7152$

S- 1 - 


\section{Table of Contents}

\begin{tabular}{|c|c|c|}
\hline S1 & Computational Methods & 3 \\
\hline $\mathbf{S 2}$ & Full citation for ref. $13 \mathrm{e}$ & $4-5$ \\
\hline Table S1 & $\begin{array}{l}\text { Energy of the intermediates and products (in } \\
\text { kcal/mol) of } \mathrm{MBH} \text { reaction for both the model and } \\
\text { real systems }\end{array}$ & 5 \\
\hline Figure S1 & $\begin{array}{l}\text { The CBS-4M optimized geometries (distances in } \AA \text { ) } \\
\text { of the intermediates and product of the MBH reaction } \\
\text { between acrolein and formaldehyde catalyzed by } \\
\mathrm{NMe}_{3} \text {. }\end{array}$ & 6 \\
\hline Figure S2 & $\begin{array}{l}\text { The } \mathrm{mPW} 1 \mathrm{~K} / 6-31+\mathrm{G}^{*} \text { optimized geometries } \\
\text { (distances in } \AA \text { ) of the intermediates and product of } \\
\text { the MBH reaction between MVK and benzaldehyde } \\
\text { catalyzed by DABCO. }\end{array}$ & 7 \\
\hline Table S2 & $\begin{array}{l}\text { Activation parameters (in } \mathrm{kcal} / \mathrm{mol} \text { ) for the water } \\
\text { assisted model } \mathrm{MBH} \text { reaction }\end{array}$ & 8 \\
\hline Figure S3 & $\begin{array}{l}\text { The mPW1K/6-31+G* optimized geometries of the } \\
\text { transition states of the MBH reaction between } \\
\text { acrylaldehyde and formaldehyde catalyzed by } \mathrm{NMe}_{3} \\
\text { through assiatance of explicit water molecules }\end{array}$ & 8 \\
\hline S3 & $\begin{array}{l}\text { The mPW1K/6-31+G* optimized coordinates and } \\
\text { total electronic energy }\left(\mathrm{E}_{\mathrm{tot}} \text { in hartree/particle) of the }\right. \\
\text { real system. }\end{array}$ & $9-18$ \\
\hline S4 & $\begin{array}{l}\text { The mPW1K/6-31+G* optimized coordinates and } \\
\text { total electronic energy }\left(\mathrm{E}_{\mathrm{tot}} \text { in hartree/particle) of the }\right. \\
\text { model system. }\end{array}$ & $18-23$ \\
\hline S5 & $\begin{array}{l}\text { The CBS- } 4 \mathrm{M} \text { optimized coordinates and the } \\
\text { electronic energy ( } \mathrm{E}_{\text {tot }} \text { in hartree/particle) of the } \\
\text { model system. }\end{array}$ & $23-27$ \\
\hline S6 & $\begin{array}{l}\text { The MP2/6-31+G* optimized coordinates and the } \\
\text { total electronic energy }\left(\mathrm{E}_{\mathrm{tot}} \text { in hartree/particle) of the }\right. \\
\text { model system. }\end{array}$ & $27-32$ \\
\hline S7 & $\begin{array}{l}\text { The mPW1K/6-31+G* optimized coordinates and } \\
\text { ground state electronic energy ( } \mathrm{E}_{\text {tot }} \text { in hartree/particle) } \\
\text { of the model systems with water molecules }\end{array}$ & $32-33$ \\
\hline
\end{tabular}




\section{S1. Computational Methods}

All the stationary points such as the transition states, reactants and products were optimized first in the gas-phase at the mPW $1 \mathrm{~K}$ level of theory in conjunction with the $6-31+\mathrm{G}^{*}$ basis set. ${ }^{1}$ The choice of mPW1K hybrid density functional is based on the reported successful applications in describing reaction barriers and other kinetic parameters. ${ }^{2}$ The calculations were repeated at the $a b$ initio MP2 as well as composite CBS-4M levels of theories. ${ }^{3}$ Further, effect of solvent is incorporated through single-point calculations at the IEF-PCM ${ }_{(\mathrm{DMSO})} / \mathrm{mPW} 1 \mathrm{~K} / 6-31+\mathrm{G}^{*} / / \mathrm{mPW} 1 \mathrm{~K} / 6-31+\mathrm{G}^{*}$ level of theory using the integral equation formalism (IEF) within the polarized continuum model (PCM) framework with UAKS radii. ${ }^{4}$ The optimized geometries have been characterized as stationary points on the potential energy surface at respective levels of theory by evaluating the corresponding Hessian indices. All the transition states were characterized by one and only one imaginary frequency pertaining to the desired reaction coordinate. The intrinsic reaction coordinate (IRC) calculations were carried out at all levels of theory (except at the CBS-4M) to further authenticate the transition states. ${ }^{5}$ The geometries of the intermediates and prereacting complex are obtained using extended IRC runs on respective transition states. The final geometries obtained from the IRC path on both sides of the first order saddle points were then subjected to further geometry optimization using stringent optimization conditions using the 'OPT=CALCFC' option. This will enable a careful walk down from the final IRC point on the PES. The Gibbs free energies in the gasphase were obtained by including zero-point vibrational energy as well as thermal corrections to the bottom-of-the-well values. All the electronic structure calculations were performed with the Gaussian03 suite of the quantum chemical programs. ${ }^{6}$ 


\section{S2. References:}

1. (a) Lynch, B. J.; Fast, P. L.; Harris, M.; Truhlar, D. G. J. Phys. Chem. A 2000, 104, 4811. (b) Lynch, B. J.; Truhlar, D. G. J. Phys. Chem. A 2001, 105, 2936. (c) Ditchfield, R.; Hehre, W. J.; Pople, J. A. J. Chem. Phys. 1971, 54, 724. (d) Hehre, W. J.; Ditchfield, R.; Pople, J. A. J. Chem. Phys. 1972, 56, 2257. (e) Gordon, M. S. Chem. Phys. Lett. 1980, 76, 163. (f) Hariharan, P. C.; Pople, J. A. Theo. Chim. Acta. 1973, 28, 213.

2. (a) Lingwood, M.; Hammond, J. R.; Hrovat, D. A.; Mayer, J. M.; Borden, W. T. J. Chem. Theory Comput. 2006, 2, 740. (b) Canepa, C. J. Phys. Chem. B 2003, 07, 4437. (c) Gilbert, T. M.; Bachrach, S. M. Organometallics 2007, 26, 2672. (d) Jones, G. O.; Guner, V. A.; Houk, K. N. J. Phys. Chem. A 2006, $110,1216$.

3. (a) Head-Gordon, M.; Pople, J. A.; Frisch, M. J. Chem. Phys. Lett. 1988, 153, 503. (b) Frisch, M. J.; Head-Gordon, M.; Pople, J. A. Chem. Phys. Lett. 1990, 166, 281. (c) Head-Gordon, M.; HeadGordon, T. Chem. Phys. Lett. 1994, 220, 122. (d) Ochterski, J. W.; Petersson, G. A.; Montgomery, J. A. Jr., J. Chem. Phys. 1996, 104, 2598. (e) Montgomery, J. A. Jr.; Frisch, M. J.; Ochterski, J. W.; Petersson, G. A. J. Chem. Phys. 2000, 112, 6532.

4. (a) Cancès, M. T.; Mennucci, B.; Tomasi, J. J. Chem. Phys. 1997, 107, 3032. (b) Mennucci, B.; Tomasi, J. J. Chem. Phys. 1997, 106, 5151. (c) Mennucci, B.; Cancès, E.; Tomasi, J. J. Phys. Chem. B 1997, 101, 10506. (d) Tomasi, J.; Mennucci, B.; Cancès, E. J. Mol. Str.: THEOCHEM 1999, 464, 211.

5. Gonzalez, C.; Schlegel, H. B. J. Chem. Phys. 1989, 90, 2154.

6. Gaussian 03, Revision C.02, Frisch, M. J.; Trucks, G. W.; Schlegel, H. B.; Scuseria, G. E.; Robb, M. A.; Cheeseman, J. R.; Montgomery, Jr., J. A.; Vreven, T.; Kudin, K. N.; Burant, J. C.; Millam, J. M.; Iyengar, S. S.; Tomasi, J.; Barone, V.; Mennucci, B.; Cossi, M.; Scalmani, G.; Rega, N.; Petersson, G. A.; Nakatsuji, H.; Hada, M.; Ehara, M.; Toyota, K.; Fukuda, R.; Hasegawa, J.; Ishida, M.; Nakajima, T.; Honda, Y.; Kitao, O.; Nakai, H.; Klene, M.; Li, X.; Knox, J. E.; Hratchian, H. P.; Cross, J. B.; Bakken, V.; Adamo, C.; Jaramillo, J.; Gomperts, R.; Stratmann, R. E.; Yazyev, O.; Austin, A. J.; Cammi, R.; Pomelli, C.; Ochterski, J. W.; Ayala, P. Y.; Morokuma, K.; Voth, G. A.; 
Salvador, P.; Dannenberg, J. J.; Zakrzewski, V. G.; Dapprich, S.; Daniels, A. D.; Strain, M. C.; Farkas, O.; Malick, D. K.; Rabuck, A. D.; Raghavachari, K.; Foresman, J. B.; Ortiz, J. V.; Cui, Q.; Baboul, A. G.; Clifford, S.; Cioslowski, J.; Stefanov, B. B.; Liu, G.; Liashenko, A.; Piskorz, P.; Komaromi, I.; Martin, R. L.; Fox, D. J.; Keith, T.; Al-Laham, M. A.; Peng, C. Y.; Nanayakkara, A.; Challacombe, M.; Gill, P. M. W.; Johnson, B.; Chen, W.; Wong, M. W.; Gonzalez, C.; and Pople, J. A.; Gaussian, Inc., Wallingford CT, 2004.

Table S1: Energy of the intermediates and products (in $\mathrm{kcal} / \mathrm{mol}$ ) in the model and real $\mathrm{MBH}$ reaction obtained at the $\mathrm{mPW} 1 \mathrm{~K} / 6-31+\mathrm{G}^{*}$ level in the gas phase $\left(\Delta \mathrm{H}^{\dagger}\right.$ and $\Delta \mathrm{G}^{\dagger}$ terms $)$ and IEF$\mathrm{PCM} / \mathrm{mPW} 1 \mathrm{~K} / 6-31+\mathrm{G}^{*} / / \mathrm{mPW} 1 \mathrm{~K} / 6-31+\mathrm{G}^{*}$ level theory in DMSO (represented as $\Delta \mathrm{E}^{\dagger} \mathrm{DMSO}$ )

\begin{tabular}{cccc}
\hline Species & $\Delta \mathrm{H}^{\dagger}$ & $\Delta \mathrm{G}^{\dagger}$ & $\Delta \mathrm{E}_{\text {DMSO }}^{\dagger}$ \\
\hline \hline $\mathbf{1 a}$ & -0.54 & 7.68 & 2.76 \\
$\mathbf{1 b}$ & 10.20 & 23.77 & -1.17 \\
$\mathbf{1 c}$ & 10.56 & 36.85 & -6.22 \\
$\mathbf{1 d}$ & -10.36 & 15.77 & -24.17 \\
$\mathbf{1 e}$ & -18.14 & -1.95 & -18.76 \\
\hline $\mathbf{2 a}$ & -1.35 & 6.37 & 3.88 \\
$\mathbf{2 b}$ & 9.62 & 23.64 & 0.36 \\
$\mathbf{2 c}$ & 15.15 & 42.81 & 9.17 \\
$\mathbf{2 c}$, & 26.61 & 54.64 & 13.99 \\
$\mathbf{2 d}$ & 7.43 & 34.50 & 2.69 \\
$\mathbf{2 e}$ & -6.63 & 15.22 & -1.05 \\
\hline \hline
\end{tabular}




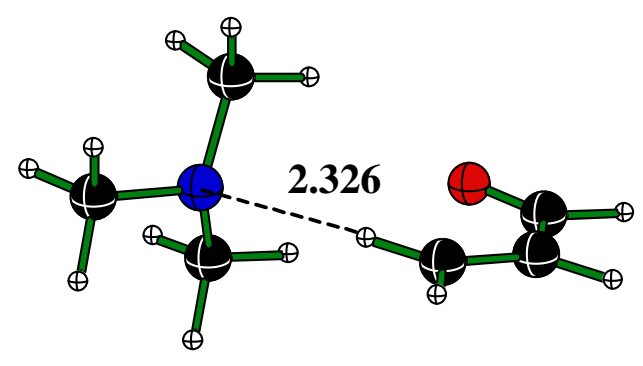

$1 a$

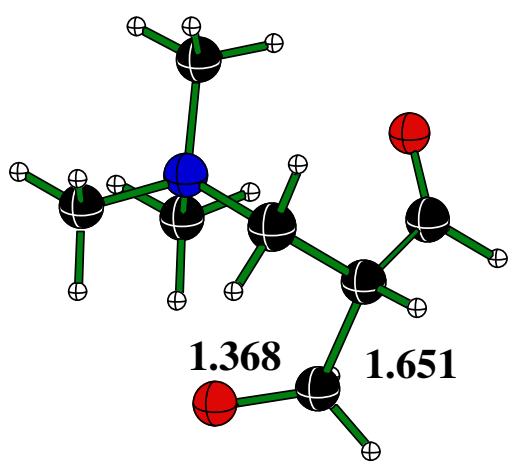

1c

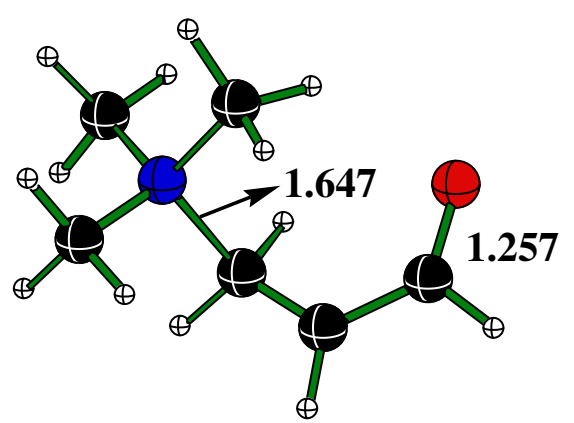

$1 b$

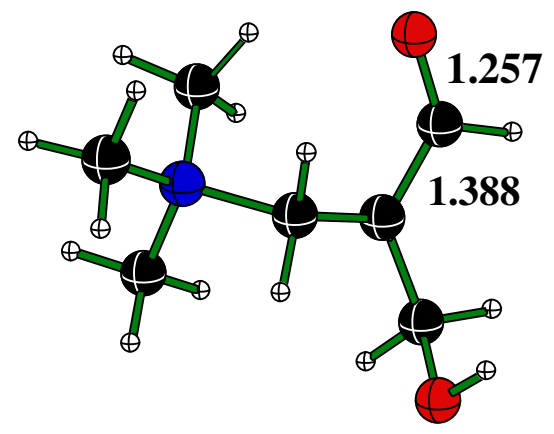

1d

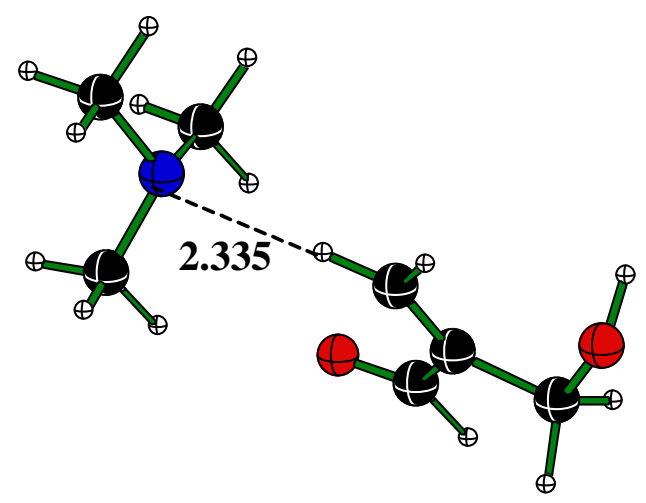

$1 \mathrm{e}$

Figure S1: The CBS-4M optimized geometries (distances in $\AA$ ) of the intermediates and product of the $\mathrm{MBH}$ reaction between acrolein and formaldehyde catalyzed by $\mathrm{NMe}_{3}$. 


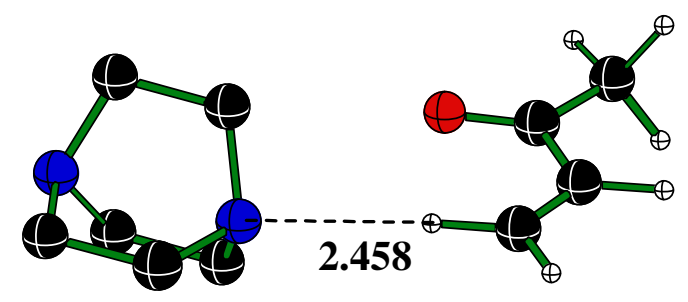

2a

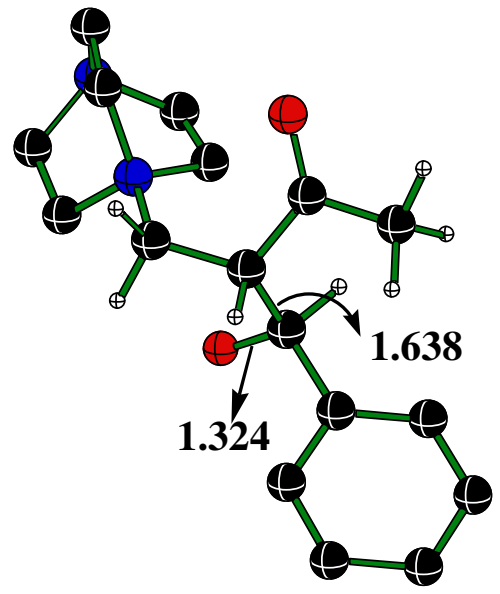

2c

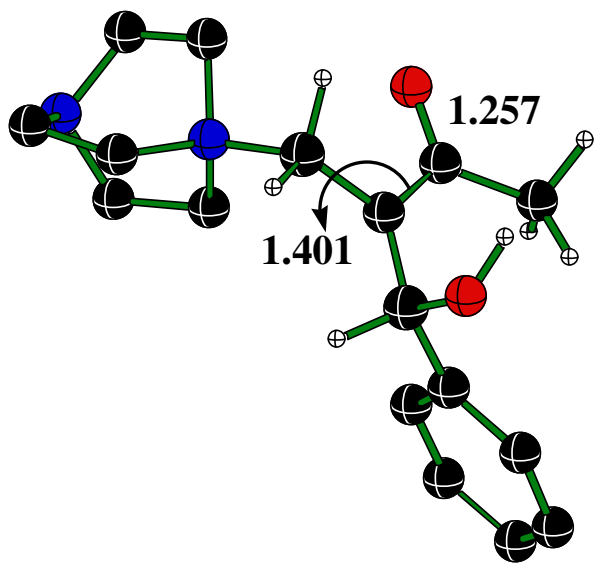

2d

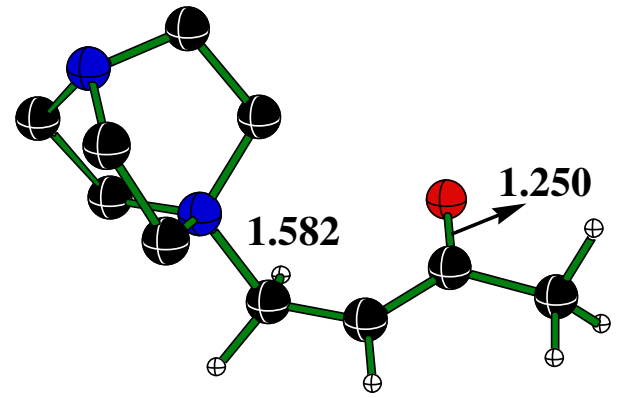

2b

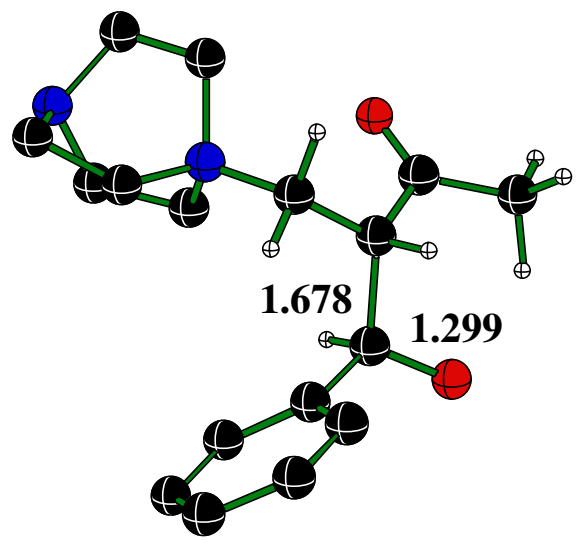

$2 c^{\prime}$

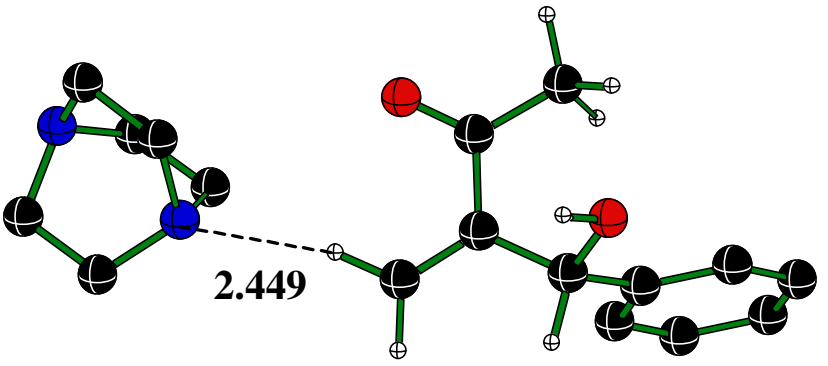

$2 e$

Figure S2: The mPW1K/6-31+G* optimized geometries (distances in $\AA$ ) of the intermediates and product of the MBH reaction between MVK and benzaldehyde catalyzed by DABCO (only selected hydrogens are shown for clarity). 
Table S2: Activation parameters (in $\mathrm{kcal} / \mathrm{mol}$ ) for the water assisted model MBH reaction obtained at the mPW1K/6-31+G* level of theory $\left(\Delta \mathrm{E}^{\dagger}, \Delta \mathrm{H}^{\dagger}\right.$, and $\Delta \mathrm{G}^{\dagger}$ terms $)$ in the gas phase and IEF$\mathrm{PCM} / \mathrm{mPW} 1 \mathrm{~K} / 6-31+\mathrm{G}^{*} / / \mathrm{mPW} 1 \mathrm{~K} / 6-31+\mathrm{G}^{*}$ in the condensed phase $\left(\Delta \mathrm{E}^{\dagger}\right.$ Water $)$

\begin{tabular}{ccccc}
\hline \hline Species & $\Delta \mathrm{E}^{\dagger}$ & $\Delta \mathrm{H}^{\dagger}$ & $\Delta \mathrm{G}^{\dagger}$ & $\Delta \mathrm{E}^{\dagger}$ Water \\
\hline $\mathbf{T S}(\mathbf{1 b}-1 \mathbf{c})_{\mathbf{2}}$ & -11.37 & -14.49 & 30.10 & -12.53 \\
$\mathbf{T S}(\mathbf{1 c - 1 d})_{2 \mathbf{W}}$ & -4.46 & -8.50 & 39.12 & -11.93 \\
\hline \hline
\end{tabular}

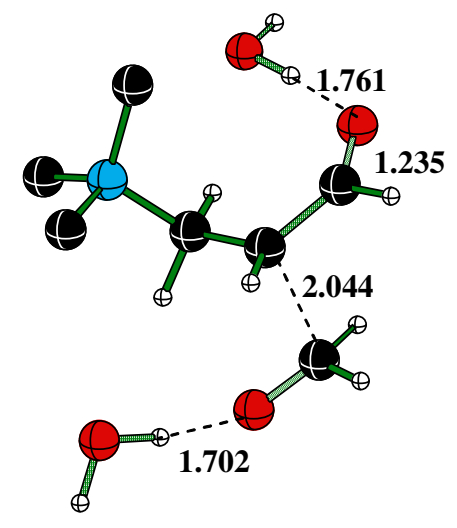

TS(1b-1c) $)_{2 W}$

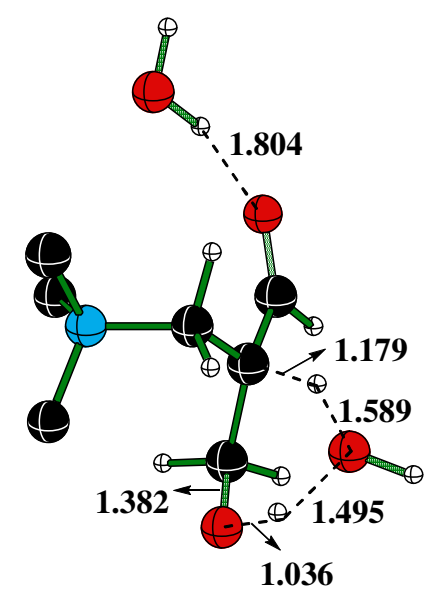

TS(1c-1d $)_{2 W}$

Figure S3: The mPW1K/6-31+G* optimized geometries (distances in $\AA$ ) of the transition states of the $\mathrm{MBH}$ reaction between acrolein and formaldehyde catalyzed by $\mathrm{NMe}_{3}$ through water assiated pathway. 
S3. The mPW1K/6-31+G* optimized coordinates, electronic energy $\left(\mathrm{E}_{\mathrm{tot}}\right.$ in hartree/particle) and number of imaginary frequencies for the real system

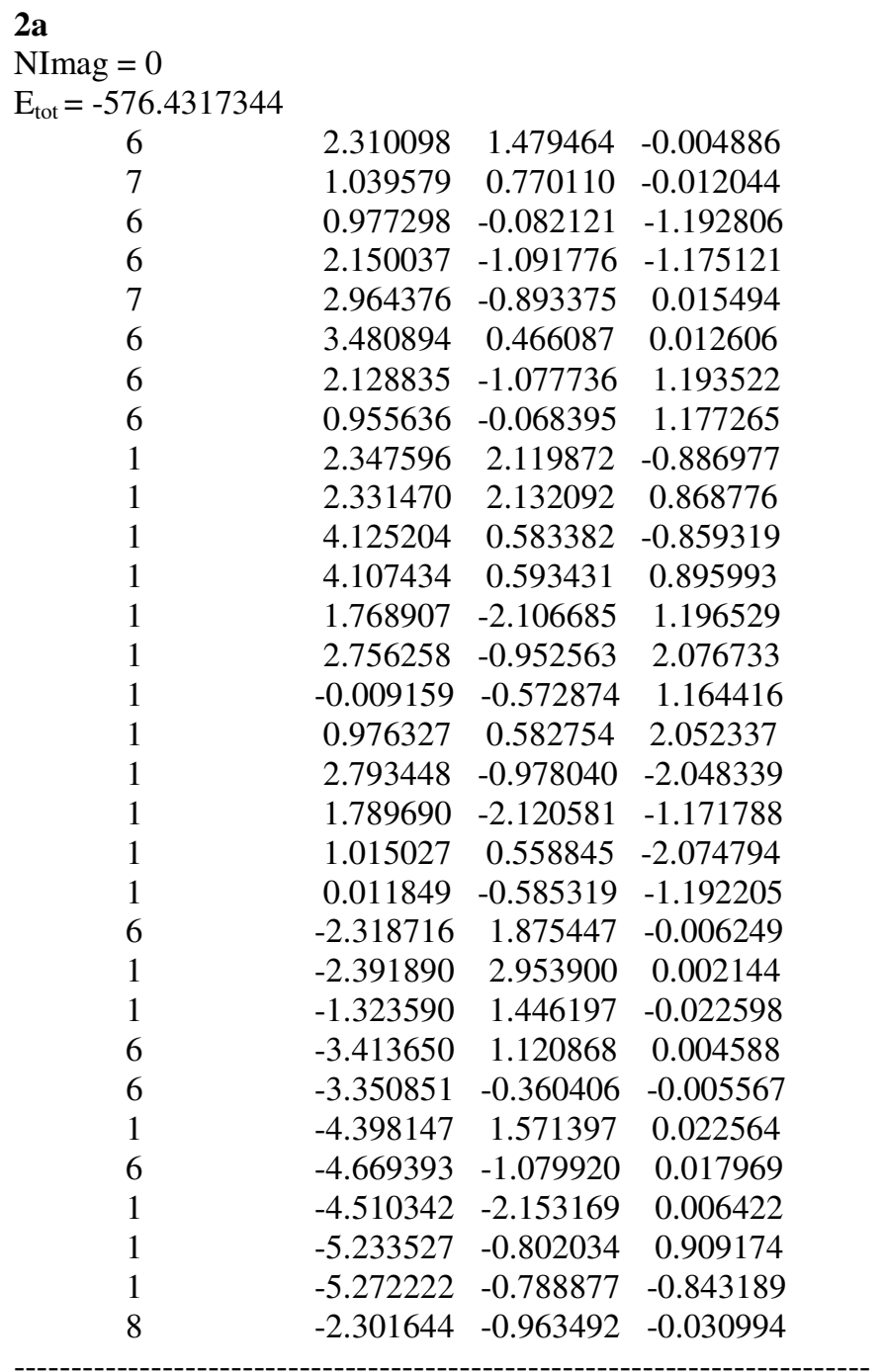

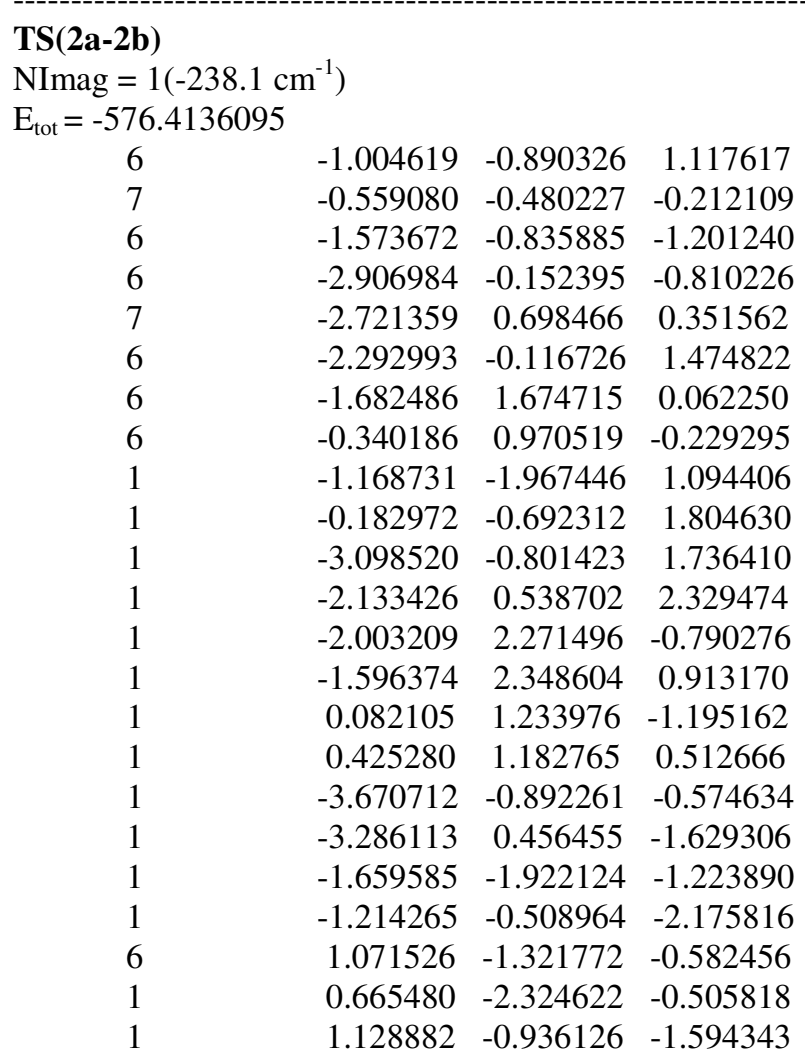




$\begin{array}{rrr}2.109051 & -0.954812 & 0.269133 \\ 2.944718 & 0.147664 & -0.080256 \\ 2.328387 & -1.540656 & 1.149945 \\ 4.185083 & 0.387413 & 0.750172 \\ 4.230675 & 1.435939 & 1.039330 \\ 4.232291 & -0.237204 & 1.640092 \\ 5.062170 & 0.185609 & 0.135675 \\ 2.720637 & 0.898944 & -1.033233\end{array}$

\section{2b}

NImag $=0$

$E_{\text {tot }}=-576.4167272$

\begin{tabular}{lrrr}
6 & -0.962995 & -1.039478 & 1.007463 \\
7 & -0.483402 & -0.467767 & -0.268977 \\
6 & -1.518266 & -0.665361 & -1.305215 \\
6 & -2.854576 & -0.100155 & -0.783503 \\
7 & -2.648248 & 0.661534 & 0.433002 \\
6 & -2.189521 & -0.235981 & 1.477541 \\
6 & -1.617767 & 1.658663 & 0.190585 \\
6 & -0.259979 & 0.992127 & -0.083240 \\
1 & -1.197780 & -2.086095 & 0.820232 \\
1 & -0.120435 & -0.994193 & 1.694041 \\
1 & -3.002245 & -0.906097 & 1.752171 \\
1 & -1.949008 & 0.360313 & 2.355618 \\
1 & -1.929995 & 2.270745 & -0.653885 \\
1 & -1.553455 & 2.314755 & 1.056503 \\
1 & 0.256594 & 1.358590 & -0.965105 \\
1 & 0.444009 & 1.080551 & 0.738502 \\
1 & -3.558443 & -0.902982 & -0.570201 \\
1 & -3.312922 & 0.543658 & -1.531651 \\
1 & -1.570268 & -1.730501 & -1.522430 \\
1 & -1.169773 & -0.155088 & -2.200211 \\
6 & 0.870084 & -1.165684 & -0.698131 \\
1 & 0.566080 & -2.207681 & -0.802390 \\
1 & 1.050456 & -0.719555 & -1.676534 \\
6 & 1.986371 & -0.949461 & 0.199125 \\
6 & 2.840543 & 0.129024 & -0.062419 \\
1 & 2.254385 & -1.712277 & 0.915073 \\
6 & 4.123474 & 0.240252 & 0.730467 \\
1 & 4.165066 & 1.212180 & 1.221112 \\
1 & 4.232834 & -0.543267 & 1.478667 \\
1 & 4.968026 & 0.193470 & 0.043515 \\
8 & 2.605529 & 1.010428 & -0.917154 \\
\hline & - & & \\
1 & & & \\
& & &
\end{tabular}

TS $(2 b-2 c)$

NImag $=1\left(-226.5 \mathrm{~cm}^{-1}\right)$

$\mathrm{E}_{\text {tot }}=-921.8847243$

$\begin{array}{lrrr}7 & 2.028106 & -0.240304 & -0.488429 \\ 6 & 0.731703 & -0.274882 & -1.255059 \\ 6 & -0.446648 & 0.534899 & -0.808182 \\ 6 & -0.408219 & 1.878053 & -0.320970 \\ 6 & -1.641560 & 2.721826 & -0.524640 \\ 6 & -1.248061 & -0.709683 & 0.632444 \\ 6 & -2.716304 & -0.581517 & 0.368999 \\ 6 & -3.344848 & -1.449598 & -0.517543 \\ 6 & -4.708329 & -1.357612 & -0.741654 \\ 6 & -5.465183 & -0.406556 & -0.068077 \\ 6 & -4.848610 & 0.447362 & 0.834069 \\ 6 & -3.480946 & 0.357855 & 1.049417 \\ 6 & 1.832025 & -0.254989 & 0.999040 \\ 6 & 2.853186 & 0.946297 & -0.864932 \\ 6 & 2.792615 & -1.469217 & -0.852774 \\ 1 & 1.022596 & -0.049607 & -2.282922\end{array}$

S- 10 - 


$$
\begin{array}{rrr}
0.404344 & -1.316342 & -1.196936 \\
-2.518672 & 2.144601 & -0.802305 \\
-1.846242 & 3.294834 & 0.377237 \\
-1.432257 & 3.438036 & -1.321154 \\
-0.930384 & -0.084193 & 1.481538 \\
-1.229704 & 0.376930 & -1.542708 \\
-2.747831 & -2.205309 & -1.007512 \\
-5.187750 & -2.036036 & -1.433759 \\
-6.530614 & -0.340225 & -0.237583 \\
-5.433031 & 1.179219 & 1.374086 \\
-3.001885 & 1.020523 & 1.758900 \\
1.112786 & -1.047212 & 1.197004 \\
1.401670 & 0.711264 & 1.241378 \\
2.223663 & 1.820189 & -0.732870 \\
3.106177 & 0.821321 & -1.916525 \\
2.783512 & -1.552634 & -1.937180 \\
2.235922 & -2.306005 & -0.439142 \\
-0.682762 & -1.817243 & 0.446123 \\
0.543490 & 2.384482 & 0.291532 \\
4.096381 & 0.976850 & 0.036454 \\
4.983460 & 1.207358 & -0.551297 \\
3.995818 & 1.750389 & 0.794741 \\
3.200533 & -0.483582 & 1.659382 \\
3.333990 & 0.210568 & 2.486537 \\
3.277443 & -1.490831 & 2.066042 \\
4.282108 & -0.296999 & 0.706675 \\
4.214902 & -1.356217 & -0.279475 \\
4.506316 & -2.297956 & 0.181667 \\
4.937525 & -1.141395 & -1.065193
\end{array}
$$

\section{TS(2b-2c')}

NImag $=1\left(-219.1 \mathrm{~cm}^{-1}\right)$

$\mathrm{E}_{\text {tot }}=-921.8780303$

$\begin{array}{lrrc}7 & -1.815606 & 0.327050 & 0.364490 \\ 6 & -1.072159 & 1.355424 & 1.168320 \\ 6 & 0.292491 & 1.818747 & 0.746488 \\ 6 & 0.649192 & 2.204153 & -0.594897 \\ 6 & 1.688893 & 3.292304 & -0.725618 \\ 6 & 1.531448 & 0.549492 & 1.662579 \\ 6 & -0.901691 & -0.751333 & -0.120940 \\ 6 & -2.541006 & 0.934238 & -0.790285 \\ 6 & -2.826541 & -0.308975 & 1.258609 \\ 1 & -1.775151 & 2.182736 & 1.276095 \\ 1 & -0.903024 & 0.888801 & 2.144548 \\ 1 & 2.348246 & 3.355262 & 0.138092 \\ 1 & 2.268512 & 3.146756 & -1.633438 \\ 1 & 1.171100 & 4.249362 & -0.813730 \\ 1 & 0.533620 & 2.615934 & 1.445066 \\ 1 & -0.323773 & -1.059795 & 0.743686 \\ 1 & -0.242431 & -0.285588 & -0.845509 \\ 1 & -1.814765 & 1.517360 & -1.346143 \\ 1 & -3.299540 & 1.588630 & -0.364145 \\ 1 & -3.383796 & 0.489571 & 1.743189 \\ 1 & -2.266014 & -0.845267 & 2.019714 \\ 8 & 0.814949 & -0.003741 & 2.544601 \\ 8 & 0.187953 & 1.709117 & -1.625641 \\ 6 & -3.149641 & -0.200671 & -1.628268 \\ 1 & -4.173732 & 0.042220 & -1.906967 \\ 1 & -2.583702 & -0.338459 & -2.546978 \\ 6 & -1.757521 & -1.877921 & -0.722501 \\ 1 & -1.356022 & -2.174604 & -1.688641 \\ 1 & -1.750166 & -2.759714 & -0.083887 \\ 7 & -3.136740 & -1.451889 & -0.893510\end{array}$

S- 11 - 


\begin{tabular}{|c|c|c|c|}
\hline & & & \\
\hline 6 & -3.721022 & -1.231966 & 0.413305 \\
\hline 1 & -3.849123 & -2.188707 & 0.915878 \\
\hline 1 & -4.711633 & -0.800362 & 0.279334 \\
\hline 1 & 2.204822 & 1.363442 & 1.974078 \\
\hline 6 & 2.229019 & -0.342725 & 0.659407 \\
\hline 6 & 2.136031 & -1.721442 & 0.823659 \\
\hline 6 & 2.811974 & -2.588583 & -0.021523 \\
\hline 6 & 3.607304 & -2.087804 & -1.041771 \\
\hline 6 & 3.732727 & -0.714259 & -1.195339 \\
\hline 6 & 3.055593 & 0.146702 & -0.346133 \\
\hline 1 & 1.545005 & -2.092260 & 1.649379 \\
\hline 1 & 2.732220 & -3.656967 & 0.127035 \\
\hline 1 & 4.138094 & -2.760040 & -1.701092 \\
\hline 1 & 4.362817 & -0.312213 & -1.976364 \\
\hline 1 & 3.181080 & 1.212487 & -0.468289 \\
\hline \multicolumn{4}{|c|}{$2 c$} \\
\hline \multirow{2}{*}{\multicolumn{4}{|c|}{$\begin{array}{l}\text { NImag }=0 \\
E_{\text {tot }}=-921.8884746\end{array}$}} \\
\hline & & & \\
\hline 7 & 1.987259 & -0.163533 & -0.474878 \\
\hline 6 & 0.725614 & 0.011611 & -1.306550 \\
\hline 6 & -0.524570 & 0.620406 & -0.708423 \\
\hline 6 & -0.429123 & 2.055466 & -0.327757 \\
\hline 6 & -1.705004 & 2.782515 & -0.022000 \\
\hline 6 & -1.128421 & -0.385218 & 0.434799 \\
\hline 6 & -2.639920 & -0.474654 & 0.270513 \\
\hline 6 & -3.176365 & -1.189366 & -0.797219 \\
\hline 6 & -4.546004 & -1.305536 & -0.960549 \\
\hline 6 & -5.411834 & -0.719922 & -0.043776 \\
\hline 6 & -4.890607 & -0.029469 & 1.037784 \\
\hline 6 & -3.514041 & 0.087412 & 1.190200 \\
\hline 6 & 1.797322 & 0.003326 & 1.005648 \\
\hline 6 & 3.040605 & 0.790303 & -0.930375 \\
\hline 6 & 2.479830 & -1.558135 & -0.702071 \\
\hline 1 & 1.039560 & 0.570365 & -2.185540 \\
\hline 1 & 0.430537 & -0.992754 & -1.585095 \\
\hline 1 & -2.536649 & 2.438400 & -0.631105 \\
\hline 1 & -1.975284 & 2.591014 & 1.016882 \\
\hline 1 & -1.550475 & 3.850958 & -0.143348 \\
\hline 1 & -0.964732 & 0.155222 & 1.396931 \\
\hline 1 & -1.241274 & 0.566515 & -1.533277 \\
\hline 1 & -2.499756 & -1.682700 & -1.481772 \\
\hline 1 & -4.943830 & -1.865386 & -1.796010 \\
\hline 1 & -6.481554 & -0.815292 & -0.166358 \\
\hline 1 & -5.553218 & 0.412116 & 1.769530 \\
\hline 1 & -3.115015 & 0.610175 & 2.050554 \\
\hline 1 & 0.963768 & -0.656766 & 1.247806 \\
\hline 1 & 1.542545 & 1.044717 & 1.161239 \\
\hline 1 & 2.605263 & 1.782486 & -0.872533 \\
\hline 1 & 3.246017 & 0.550367 & -1.971907 \\
\hline 1 & 2.495862 & -1.720746 & -1.777334 \\
\hline 1 & 1.717850 & -2.197626 & -0.261333 \\
\hline 8 & -0.511622 & -1.553402 & 0.352139 \\
\hline 8 & 0.640330 & 2.633098 & -0.230087 \\
\hline 6 & 4.287986 & 0.618091 & -0.046545 \\
\hline 1 & 5.181102 & 0.553269 & -0.665863 \\
\hline 1 & 4.411384 & 1.474612 & 0.613209 \\
\hline 6 & 3.100632 & -0.396504 & 1.715657 \\
\hline 1 & 3.382906 & 0.365033 & 2.440229 \\
\hline 1 & 2.968468 & -1.329799 & 2.259911 \\
\hline 7 & 4.187687 & -0.573728 & 0.771164 \\
\hline 6 & 3.876382 & -1.707372 & -0.076891 \\
\hline 1 & 3.923964 & -2.618052 & 0.516687 \\
\hline 1 & 4.643389 & -1.780586 & -0.846587 \\
\hline
\end{tabular}

S- 12 - 
2c'

NImag $=0$

$\mathrm{E}_{\text {tot }}=-921.869287$

$\begin{array}{lrrr}7 & 1.385452 & -0.229899 & -0.493116 \\ 6 & 0.203174 & 0.444818 & -1.165522 \\ 6 & -0.600651 & 1.545579 & -0.486111 \\ 6 & 0.143720 & 2.780953 & -0.119182 \\ 6 & -0.660450 & 4.026573 & -0.032947 \\ 6 & -1.694422 & 1.161157 & 0.727297 \\ 6 & 1.345099 & -0.160476 & 0.999716 \\ 6 & 2.677518 & 0.360597 & -0.967252 \\ 6 & 1.376570 & -1.671964 & -0.885176 \\ 1 & 0.606346 & 0.800841 & -2.113264 \\ 1 & -0.493175 & -0.358863 & -1.387062 \\ 1 & -0.978275 & 4.332310 & -1.031103 \\ 1 & -1.580175 & 3.766927 & 0.512279 \\ 1 & -0.085295 & 4.822891 & 0.430528 \\ 1 & -1.331793 & 1.811342 & -1.252704 \\ 1 & 0.354968 & -0.475002 & 1.301804 \\ 1 & 1.477200 & 0.885188 & 1.253216 \\ 1 & 2.598903 & 1.432252 & -0.821641 \\ 1 & 2.739918 & 0.136677 & -2.030284 \\ 1 & 1.254856 & -1.713809 & -1.964942 \\ 1 & 0.493916 & -2.110429 & -0.425021 \\ 8 & -2.600228 & 2.088206 & 0.819205 \\ 8 & 1.339797 & 2.761107 & 0.151159 \\ 6 & 3.828511 & -0.274752 & -0.173275 \\ 1 & 4.647410 & -0.534095 & -0.841865 \\ 1 & 4.217779 & 0.425308 & 0.562868 \\ 6 & 2.450591 & -1.069116 & 1.563850 \\ 1 & 2.994694 & -0.547063 & 2.347856 \\ 1 & 2.028321 & -1.969693 & 2.006412 \\ 7 & 3.380159 & -1.465739 & 0.522673 \\ 6 & 2.687356 & -2.325848 & -0.414482 \\ 1 & 2.476626 & -3.281237 & 0.061718 \\ 1 & 3.349121 & -2.521011 & -1.256355 \\ 6 & -2.263071 & -0.212176 & 0.363329 \\ 6 & -3.083798 & -0.345693 & -0.755267 \\ 6 & -3.628721 & -1.570772 & -1.099326 \\ 6 & -3.385561 & -2.693344 & -0.313562 \\ 6 & -2.610676 & -2.566763 & 0.827231 \\ 6 & -2.056177 & -1.333584 & 1.157672 \\ 1 & -3.317208 & 0.539516 & -1.331069 \\ 1 & -4.262422 & -1.652519 & -1.971976 \\ 1 & -3.822485 & -3.646977 & -0.574423 \\ 1 & -2.453472 & -3.420274 & 1.473162 \\ 1 & -1.495327 & -1.234644 & 2.080006 \\ 1 & -1.070337 & 1.016188 & 1.640083\end{array}$

TS(2c-2d)

NImag $=1\left(-1601.7 \mathrm{~cm}^{-1}\right)$

$\mathrm{E}_{\text {tot }}=-921.8341292$

$\begin{array}{lrrr}7 & 2.077691 & -0.297410 & 0.372476 \\ 6 & 1.024573 & -1.346397 & 0.805117 \\ 6 & -0.367541 & -1.269983 & 0.276954 \\ 6 & -0.556988 & -1.593652 & -1.129998 \\ 6 & -1.947012 & -1.755158 & -1.673698 \\ 6 & -1.357419 & -0.500222 & 1.196454 \\ 6 & -2.490129 & 0.277721 & 0.548836 \\ 6 & -3.795462 & -0.072458 & 0.866572 \\ 6 & -4.868266 & 0.635094 & 0.342883 \\ 6 & -4.652132 & 1.711507 & -0.504698 \\ 6 & -3.350884 & 2.078606 & -0.819333\end{array}$

S- 13 - 


\begin{tabular}{|c|c|c|c|}
\hline 6 & -2.283167 & 1.369010 & -0.290983 \\
\hline 6 & 1.452607 & 0.929652 & -0.188615 \\
\hline 6 & 2.996102 & -0.877550 & -0.655527 \\
\hline 6 & 2.899021 & 0.091105 & 1.551208 \\
\hline 1 & 1.490479 & -2.293899 & 0.547717 \\
\hline 1 & 0.972677 & -1.264251 & 1.888625 \\
\hline 1 & -2.651446 & -2.080069 & -0.913341 \\
\hline 1 & -2.306285 & -0.801787 & -2.060822 \\
\hline 1 & -1.912656 & -2.467343 & -2.494263 \\
\hline 1 & -0.775541 & 0.257543 & 1.763629 \\
\hline 1 & -0.963572 & -2.129287 & 1.013696 \\
\hline 1 & -3.930230 & -0.910611 & 1.534781 \\
\hline 1 & -5.878176 & 0.346905 & 0.600837 \\
\hline 1 & -5.487036 & 2.262929 & -0.913726 \\
\hline 1 & -3.168521 & 2.918358 & -1.476139 \\
\hline 1 & -1.276988 & 1.670733 & -0.551997 \\
\hline 1 & 0.690892 & 1.254886 & 0.511963 \\
\hline 1 & 0.966701 & 0.628981 & -1.111198 \\
\hline 1 & 2.359738 & -1.316472 & -1.419044 \\
\hline 1 & 3.544796 & -1.672629 & -0.154757 \\
\hline 1 & 3.227211 & -0.825622 & 2.035052 \\
\hline 1 & 2.237433 & 0.616010 & 2.236603 \\
\hline 8 & -1.776237 & -1.541098 & 1.997593 \\
\hline 8 & 0.403112 & -1.825736 & -1.865087 \\
\hline 6 & 3.926046 & 0.233669 & -1.166684 \\
\hline 1 & 4.951900 & -0.126072 & -1.218766 \\
\hline 1 & 3.640571 & 0.545780 & -2.169223 \\
\hline 6 & 2.550112 & 1.987335 & -0.403996 \\
\hline 1 & 2.443444 & 2.442298 & -1.386407 \\
\hline 1 & 2.471083 & 2.784949 & 0.332657 \\
\hline 7 & 3.868628 & 1.393194 & -0.295091 \\
\hline 6 & 4.074693 & 0.963679 & 1.073228 \\
\hline 1 & 4.177078 & 1.837868 & 1.713076 \\
\hline 1 & 5.012285 & 0.413239 & 1.122993 \\
\hline
\end{tabular}

\section{TS(2c'-2d)}

$\mathrm{NImag}=1\left(-1527.5 \mathrm{~cm}^{-1}\right)$

$\mathrm{E}_{\text {tot }}=-921.8412435$

$\begin{array}{lrrr}7 & 1.296831 & -0.026833 & -0.536381 \\ 6 & 0.209833 & 0.835595 & -1.229894 \\ 6 & -0.665702 & 1.702407 & -0.398553 \\ 6 & -0.015938 & 2.848047 & 0.220556 \\ 6 & -0.859448 & 3.800792 & 1.020665 \\ 6 & -2.060280 & 1.209310 & 0.092269 \\ 6 & 1.292518 & 0.107074 & 0.949162 \\ 6 & 2.644165 & 0.395817 & -1.018189 \\ 6 & 1.096258 & -1.462934 & -0.888085 \\ 1 & 0.768992 & 1.449714 & -1.928900 \\ 1 & -0.396158 & 0.129612 & -1.788851 \\ 1 & -1.881007 & 3.841785 & 0.649030 \\ 1 & -0.893410 & 3.474791 & 2.061551 \\ 1 & -0.400541 & 4.785214 & 0.993161 \\ 1 & -1.532190 & 2.190870 & -1.186873 \\ 1 & 0.274731 & -0.048436 & 1.283809 \\ 1 & 1.585011 & 1.129557 & 1.158419 \\ 1 & 2.699028 & 1.469900 & -0.862925 \\ 1 & 2.671425 & 0.189871 & -2.086173 \\ 1 & 0.986380 & -1.517354 & -1.967947 \\ 1 & 0.156649 & -1.770165 & -0.435354 \\ 8 & -2.858768 & 1.793068 & -0.867124 \\ 8 & 1.179706 & 3.082152 & 0.047040 \\ 6 & 3.717950 & -0.390605 & -0.243863 \\ 1 & 4.460325 & -0.793839 & -0.930018 \\ 1 & 4.240286 & 0.260063 & 0.454488\end{array}$

S- 14 - 


$\begin{array}{rrr}2.268388 & -0.923972 & 1.538790 \\ 2.887229 & -0.455922 & 2.301762 \\ 1.729580 & -1.742879 & 2.011954 \\ 3.122110 & -1.479826 & 0.506196 \\ 2.303166 & -2.275556 & -0.385411 \\ 1.961312 & -3.164383 & 0.140584 \\ 2.922942 & -2.607204 & -1.216817 \\ -2.210558 & -0.297606 & 0.174115 \\ -2.635586 & -1.004850 & -0.947124 \\ -2.756229 & -2.385996 & -0.913978 \\ -2.474175 & -3.087793 & 0.252076 \\ -2.095249 & -2.390365 & 1.390749 \\ -1.972680 & -1.007120 & 1.348738 \\ -2.902603 & -0.430860 & -1.823730 \\ -3.092766 & -2.918001 & -1.793497 \\ -2.582770 & -4.162905 & 0.282569 \\ -1.919675 & -2.920404 & 2.317174 \\ -1.720460 & -0.467523 & 2.254529 \\ -2.240995 & 1.589873 & 1.111891\end{array}$

2d

NImag $=0$

$\mathrm{E}_{\text {tot }}=-921.9007087$

$\begin{array}{lrrr}7 & -2.102174 & -0.236009 & -0.412121 \\ 6 & -0.934746 & -1.235884 & -0.823694 \\ 6 & 0.378699 & -1.056914 & -0.194753 \\ 6 & 0.480935 & -1.524936 & 1.122271 \\ 6 & 1.808657 & -1.726163 & 1.813356 \\ 6 & 1.497451 & -0.647684 & -1.101087 \\ 6 & 2.616190 & 0.188635 & -0.500248 \\ 6 & 3.933166 & -0.006922 & -0.900337 \\ 6 & 4.951769 & 0.801964 & -0.415558 \\ 6 & 4.670228 & 1.830175 & 0.469934 \\ 6 & 3.357846 & 2.039370 & 0.869117 \\ 6 & 2.344781 & 1.224837 & 0.388881 \\ 6 & -1.576368 & 0.951807 & 0.303428 \\ 6 & -3.071868 & -0.936961 & 0.479847 \\ 6 & -2.832909 & 0.219554 & -1.618021 \\ 1 & -1.389041 & -2.197019 & -0.588265 \\ 1 & -0.873733 & -1.122422 & -1.903842 \\ 1 & 2.668219 & -1.741073 & 1.149666 \\ 1 & 1.966512 & -0.935245 & 2.546774 \\ 1 & 1.752568 & -2.665662 & 2.360263 \\ 1 & 1.068199 & -0.017602 & -1.887604 \\ 1 & 1.910399 & -2.526607 & -1.286422 \\ 1 & 4.149443 & -0.804448 & -1.594202 \\ 1 & 5.969849 & 0.627064 & -0.734930 \\ 1 & 5.463041 & 2.459870 & 0.848107 \\ 1 & 3.123602 & 2.832433 & 1.565899 \\ 1 & 1.330879 & 1.378077 & 0.729219 \\ 1 & -0.772054 & 1.365115 & -0.296914 \\ 1 & -1.149641 & 0.577648 & 1.228912 \\ 1 & -2.469456 & -1.407701 & 1.255490 \\ 1 & -3.538999 & -1.710644 & -0.126640 \\ 1 & -3.087842 & -0.662665 & -2.201101 \\ 1 & -2.141189 & 0.822428 & -2.203018 \\ 8 & 2.047673 & -1.749624 & -1.830723 \\ 8 & -0.544135 & -1.801227 & 1.795149 \\ 6 & -4.094908 & 0.086610 & 0.994652 \\ 1 & -5.104917 & -0.312439 & 0.918464 \\ 1 & -3.915926 & 0.317250 & 2.043002 \\ 6 & -2.731538 & 1.946371 & 0.517301 \\ 1 & -2.729602 & 2.307333 & 1.543770 \\ 1 & -2.625722 & 2.815206 & -0.130827 \\ & & & \end{array}$

S- 15 - 


$\begin{array}{llll}7 & -4.012725 & 1.324205 & 0.237587 \\ 6 & -4.078403 & 1.013941 & -1.176130 \\ 1 & -4.155214 & 1.939920 & -1.743099 \\ 1 & -4.987269 & 0.443833 & -1.360136\end{array}$

$\begin{array}{crrr}\text { TS(2d-2e) } & & & \\ \text { NImag = } 1\left(-217.0 \mathrm{~cm}^{-1}\right) & & & \\ \mathrm{E}_{\text {tot }}=-921.8975697 & & & \\ 7 & -2.139329 & 0.218680 & 0.413617 \\ 6 & -0.803096 & 1.424166 & 0.876449 \\ 6 & 0.461226 & 1.253415 & 0.286928 \\ 6 & 0.611394 & 1.803191 & -1.029717 \\ 6 & 1.981447 & 2.042436 & -1.612675 \\ 6 & 1.583897 & 0.709336 & 1.122236 \\ 6 & 2.554246 & -0.261717 & 0.477526 \\ 6 & 3.866807 & -0.334634 & 0.936507 \\ 6 & 4.751734 & -1.264839 & 0.412821 \\ 6 & 4.342049 & -2.143295 & -0.579125 \\ 6 & 3.038532 & -2.076972 & -1.045316 \\ 6 & 2.156196 & -1.144076 & -0.520215 \\ 6 & -1.562089 & -0.918015 & -0.303565 \\ 6 & -3.094830 & 0.911003 & -0.466484 \\ 6 & -2.840642 & -0.262895 & 1.606233 \\ 1 & -1.379240 & 2.272344 & 0.527940 \\ 1 & -0.866640 & 1.261404 & 1.947703 \\ 1 & 2.769712 & 2.082298 & -0.866734 \\ 1 & 2.220740 & 1.246511 & -2.317993 \\ 1 & 1.949532 & 2.974958 & -2.172192 \\ 1 & 1.116008 & 0.155746 & 1.946976 \\ 1 & 1.812624 & 2.526876 & 1.735356 \\ 1 & 4.192529 & 0.354078 & 1.700181 \\ 1 & 5.767672 & -1.300544 & 0.780747 \\ 1 & 5.032903 & -2.866091 & -0.989355 \\ 1 & 2.707819 & -2.745233 & -1.828341 \\ 1 & 1.152200 & -1.084564 & -0.911543 \\ 1 & -0.780421 & -1.344720 & 0.320752 \\ 1 & -1.091718 & -0.518426 & -1.198008 \\ 1 & -2.516123 & 1.364781 & -1.268051 \\ 1 & -3.547601 & 1.712679 & 0.115878 \\ 1 & -3.166483 & 0.603413 & 2.180805 \\ 1 & -2.122904 & -0.814135 & 2.212063 \\ 8 & 2.367831 & 1.746836 & 1.714199 \\ 8 & -0.375470 & 2.100674 & -1.714354 \\ 6 & -4.147628 & -0.106746 & -0.953963 \\ 1 & -5.157544 & 0.259832 & -0.776268 \\ 1 & -4.050932 & -0.282777 & -2.023806 \\ 6 & -2.686424 & -1.930026 & -0.617206 \\ 1 & -2.689965 & -2.187232 & -1.674871 \\ 1 & -2.548095 & -2.855426 & -0.059746 \\ 7 & -3.984366 & -1.377647 & -0.268272 \\ 6 & -4.028454 & -1.149453 & 1.163349 \\ 1 & -4.003729 & -2.110667 & 1.674314 \\ 1 & -4.979563 & -0.677702 & 1.405320\end{array}$

\begin{tabular}{|c|c|c|c|}
\hline \multicolumn{4}{|c|}{$\begin{array}{l}\mathbf{2 e} \\
\text { NImag }=0 \\
E_{\text {tot }}=-921.9206799\end{array}$} \\
\hline 7 & -3.173972 & 0.186280 & -0.675184 \\
\hline 6 & 0.223690 & -0.414509 & -1.363278 \\
\hline 6 & 1.211891 & -0.765200 & -0.541064 \\
\hline 6 & 0.882517 & -1.015635 & 0.904431 \\
\hline 6 & 1.992845 & -1.151178 & 1.901532 \\
\hline 6 & 2.617662 & -0.936632 & -1.065394 \\
\hline 6 & 3.619715 & 0.051055 & -0.512410 \\
\hline
\end{tabular}




$\begin{array}{lrrr}6 & 4.891606 & -0.357772 & -0.132376 \\ 6 & 5.814496 & 0.567611 & 0.334035 \\ 6 & 5.479640 & 1.909842 & 0.420782 \\ 6 & 4.212734 & 2.323814 & 0.035367 \\ 6 & 3.289790 & 1.399984 & -0.428334 \\ 6 & -2.986080 & 1.094424 & 0.449886 \\ 6 & -3.575435 & -1.117985 & -0.161880 \\ 6 & -4.229543 & 0.704611 & -1.532317 \\ 1 & -0.797159 & -0.289393 & -1.024379 \\ 1 & 0.419438 & -0.231596 & -2.412243 \\ 1 & 2.731409 & -1.875895 & 1.566430 \\ 1 & 2.510867 & -0.198935 & 2.013883 \\ 1 & 1.571271 & -1.448161 & 2.856878 \\ 1 & 2.571692 & -0.769915 & -2.146534 \\ 1 & 2.506187 & -2.862584 & -1.255170 \\ 1 & 5.151091 & -1.402695 & -0.198419 \\ 1 & 6.799373 & 0.235608 & 0.630621 \\ 1 & 6.199189 & 2.628635 & 0.785658 \\ 1 & 3.940329 & 3.367741 & 0.098074 \\ 1 & 2.302084 & 1.729946 & -0.720184 \\ 1 & -2.644855 & 2.054025 & 0.058622 \\ 1 & -2.190021 & 0.691869 & 1.074242 \\ 1 & -2.757709 & -1.506160 & 0.443018 \\ 1 & -3.703804 & -1.789583 & -1.011917 \\ 1 & -4.332592 & 0.037113 & -2.388704 \\ 1 & -3.910925 & 1.674772 & -1.915688 \\ 8 & 3.097237 & -2.242681 & -0.825350 \\ 8 & -0.273925 & -1.095661 & 1.253861 \\ 6 & -4.883557 & -0.981687 & 0.654768 \\ 1 & -5.679771 & -1.605986 & 0.247779 \\ 1 & -4.737609 & -1.280650 & 1.692819 \\ 6 & -4.311452 & 1.241486 & 1.235834 \\ 1 & -4.191139 & 0.947741 & 2.278683 \\ 1 & -4.671289 & 2.270942 & 1.229702 \\ 7 & -5.339974 & 0.400373 & 0.642436 \\ 6 & -5.552283 & 0.814921 & -0.734700 \\ 1 & -5.932714 & 1.836890 & -0.728800 \\ 1 & -6.331625 & 0.183529 & -1.162522 \\ ------------------------------------------------ \\ 1 & --19 & \end{array}$

\section{DABCO}

$\mathrm{NImag}=0$

$E_{\text {tot }}=-345.2600648$

$\begin{array}{rrrr}6 & -0.774489 & -0.912444 & -1.018343 \\ 7 & -1.272897 & -0.000152 & 0.000278 \\ 6 & -0.773944 & -0.426282 & 1.299414 \\ 6 & 0.774809 & -0.425696 & 1.299117 \\ 7 & 1.272949 & 0.000211 & -0.000451 \\ 6 & 0.774138 & -0.912530 & -1.018545 \\ 6 & 0.774040 & 1.338587 & -0.281209 \\ 6 & -0.774619 & 1.338424 & -0.280393 \\ 1 & -1.175705 & -1.904584 & -0.809601 \\ 1 & -1.175969 & -0.596342 & -1.981538 \\ 1 & 1.175339 & -1.904561 & -0.809278 \\ 1 & 1.175471 & -0.596888 & -1.981949 \\ 1 & 1.175629 & 2.014828 & 0.473986 \\ 1 & 1.174857 & 1.653651 & -1.245071 \\ 1 & -1.175486 & 2.014236 & 0.475577 \\ 1 & -1.176523 & 1.654057 & -1.243617 \\ 1 & 1.176492 & -1.417792 & 1.507064 \\ 1 & 1.176030 & 0.251414 & 2.053732 \\ 1 & -1.174732 & -1.418815 & 1.507019 \\ 1 & -1.175372 & 0.250030 & 2.054636\end{array}$




\begin{tabular}{|c|c|c|c|}
\hline $\begin{array}{l}\text { NImag }=0 \\
E_{\text {tot }}=-231.1674247\end{array}$ & & & \\
\hline 6 & -1.999643 & -0.100581 & 0.000062 \\
\hline 1 & -2.904095 & -0.690530 & 0.000054 \\
\hline 1 & -2.094827 & 0.976109 & 0.000205 \\
\hline 6 & -0.796630 & -0.662352 & -0.000082 \\
\hline 6 & 0.440336 & 0.162386 & -0.000031 \\
\hline 1 & -0.682312 & -1.738697 & -0.000208 \\
\hline 6 & 1.740124 & -0.589470 & 0.000060 \\
\hline 1 & 2.573682 & 0.104974 & 0.000204 \\
\hline 1 & 1.800468 & -1.235782 & 0.876649 \\
\hline 1 & 1.800658 & -1.235623 & -0.876636 \\
\hline 8 & 0.400164 & 1.369957 & -0.000040 \\
\hline
\end{tabular}

\section{PhCHO}

NImag $=0$

$\mathrm{E}_{\mathrm{tot}}=-345.4763897$

$\begin{array}{lrrr}6 & -1.721270 & 1.056635 & 0.000044 \\ 6 & -0.353675 & 1.281409 & -0.000079 \\ 6 & 0.529736 & 0.208127 & -0.000146 \\ 6 & 0.042628 & -1.096777 & -0.000083 \\ 6 & -1.321230 & -1.320470 & 0.000040 \\ 6 & -2.202299 & -0.244123 & 0.000104 \\ 1 & -2.408677 & 1.889962 & 0.000095 \\ 1 & 0.031833 & 2.292782 & -0.000129 \\ 1 & 0.747346 & -1.915420 & -0.000136 \\ 1 & -1.704167 & -2.330736 & 0.000084 \\ 1 & -3.268178 & -0.422693 & 0.000201 \\ 6 & 1.980094 & 0.463608 & -0.000297 \\ 8 & 2.826580 & -0.391758 & 0.000265 \\ 1 & 2.265296 & 1.529714 & 0.000263\end{array}$

S4. The mPW1K/6-31+G* optimized coordinates, electronic energy ( $\mathrm{E}_{\text {tot }}$ in hartree/particle) and number of imaginary frequencies of the model system.

$\begin{array}{crrr}\mathbf{1 a} & & & \\ \text { NImag = } 0 & & & \\ \mathrm{E}_{\text {tot }}=-366.2846354 & & & \\ 6 & 1.495974 & -0.593076 & 1.237412 \\ 7 & 1.639412 & 0.104780 & -0.016252 \\ 6 & 2.832962 & 0.909400 & -0.036945 \\ 6 & 1.603959 & -0.813965 & -1.127370 \\ 1 & 1.471776 & 0.122630 & 2.057874 \\ 1 & 0.562198 & -1.150349 & 1.239838 \\ 1 & 1.655135 & -0.262225 & -2.064754 \\ 1 & 0.672020 & -1.373796 & -1.108900 \\ 1 & 2.810462 & 1.628562 & 0.780265 \\ 1 & 2.892157 & 1.462426 & -0.972912 \\ 6 & -1.514801 & 1.527515 & -0.081431 \\ 1 & -1.484109 & 2.607154 & -0.112707 \\ 1 & -0.565332 & 1.002676 & -0.096288 \\ 6 & -2.678315 & 0.883575 & -0.026139 \\ 6 & -2.759112 & -0.585246 & 0.018236 \\ 1 & -3.618459 & 1.419154 & -0.011343 \\ 8 & -1.811445 & -1.330173 & 0.006875 \\ 1 & 2.322169 & -1.296238 & 1.423782 \\ 1 & 2.440655 & -1.528943 & -1.106818 \\ 1 & 3.750658 & 0.309284 & 0.062803 \\ 1 & -3.777649 & -1.001635 & 0.065340\end{array}$

TS(1a-1b)

NImag $=1\left(-268.8 \mathrm{~cm}^{-1}\right)$ 


$\begin{array}{crrr}\mathrm{E}_{\text {tot }}=-366.2692097 & & & \\ 6 & -1.681677 & 0.985942 & -0.868801 \\ 7 & -1.140721 & -0.075769 & -0.036817 \\ 6 & -2.118066 & -0.576931 & 0.914419 \\ 6 & -0.586135 & -1.144374 & -0.860305 \\ 1 & -2.110649 & 1.761979 & -0.239179 \\ 1 & -0.865486 & 1.410152 & -1.449635 \\ 1 & -0.142441 & -1.902241 & -0.223341 \\ 1 & 0.202933 & -0.726173 & -1.477439 \\ 1 & -2.456625 & 0.238112 & 1.550297 \\ 1 & -1.654719 & -1.339509 & 1.535437 \\ 6 & 0.258195 & 0.647569 & 0.908278 \\ 1 & -0.339338 & 1.393139 & 1.422816 \\ 1 & 0.451645 & -0.242618 & 1.497838 \\ 6 & 1.325798 & 1.085061 & 0.118265 \\ 6 & 2.395464 & 0.198258 & -0.163187 \\ 1 & 1.395126 & 2.114673 & -0.202567 \\ 8 & 2.433687 & -1.002196 & 0.105028 \\ 1 & -2.450327 & 0.603450 & -1.541353 \\ 1 & -1.363566 & -1.581204 & -1.489104 \\ 1 & -2.979800 & -1.010633 & 0.404806 \\ 1 & 3.267320 & 0.655676 & -0.663107\end{array}$

\section{$1 \mathrm{~b}$}

NImag $=0$

$E_{\text {tot }}=-366.2699215$

$\begin{array}{lrrr}6 & 1.629822 & 1.027931 & 0.819412 \\ 7 & 1.051921 & -0.059254 & 0.020588 \\ 6 & 2.066973 & -0.657226 & -0.852794 \\ 6 & 0.481075 & -1.082837 & 0.916880 \\ 1 & 2.092702 & 1.753229 & 0.156378 \\ 1 & 0.818403 & 1.499893 & 1.365826 \\ 1 & -0.013087 & -1.841545 & 0.321709 \\ 1 & -0.271479 & -0.603798 & 1.531901 \\ 1 & 2.444545 & 0.100142 & -1.533626 \\ 1 & 1.610302 & -1.459425 & -1.424016 \\ 6 & -0.124886 & 0.543396 & -0.886165 \\ 1 & 0.415677 & 1.262496 & -1.500681 \\ 1 & -0.411001 & -0.327909 & -1.474348 \\ 6 & -1.244954 & 1.085107 & -0.153307 \\ 6 & -2.328365 & 0.242855 & 0.110700 \\ 1 & -1.328482 & 2.149160 & 0.010848 \\ 8 & -2.363960 & -0.984797 & -0.078524 \\ 1 & 2.371976 & 0.629215 & 1.505922 \\ 1 & 1.277216 & -1.504782 & 1.525673 \\ 1 & 2.886355 & -1.054156 & -0.259147 \\ 1 & -3.222883 & 0.735280 & 0.529285\end{array}$

TS(1b-1c)

NImag $=1\left(-184.4 \mathrm{~cm}^{-1}\right)$

$E_{\text {tot }}=-480.7289571$

$\begin{array}{lrrr}6 & -1.648064 & -1.100142 & 1.117797 \\ 7 & -1.452696 & -0.148880 & 0.002105 \\ 6 & -2.346763 & -0.523561 & -1.113099 \\ 6 & -1.767392 & 1.225721 & 0.446041 \\ 1 & -1.356854 & -2.091825 & 0.787532 \\ 1 & -1.017745 & -0.806303 & 1.947987 \\ 1 & -1.537275 & 1.915830 & -0.358319 \\ 1 & -1.143729 & 1.467163 & 1.298410 \\ 1 & -2.076903 & -1.516024 & -1.459125 \\ 1 & -2.217499 & 0.189543 & -1.920654 \\ 6 & -0.018284 & -0.236240 & -0.503696 \\ 1 & 0.146973 & -1.289250 & -0.755096 \\ 1 & 0.011816 & 0.387614 & -1.394366\end{array}$




\begin{tabular}{lrrr}
6 & 1.050490 & 0.177214 & 0.432303 \\
6 & 1.471066 & 1.557472 & 0.358989 \\
8 & 1.146348 & 2.357199 & -0.504534 \\
1 & -2.691591 & -1.084849 & 1.419604 \\
1 & -2.817705 & 1.280668 & 0.719855 \\
1 & -3.378511 & -0.514509 & -0.773387 \\
1 & 2.204427 & 1.865390 & 1.123460 \\
1 & 0.998661 & -0.217037 & 1.440876 \\
6 & 2.394461 & -0.960150 & -0.108243 \\
1 & 2.708164 & -0.458513 & -1.043661 \\
1 & 3.125722 & -0.744391 & 0.689862 \\
8 & 1.903383 & -2.130103 & -0.185249 \\
\hline & &
\end{tabular}

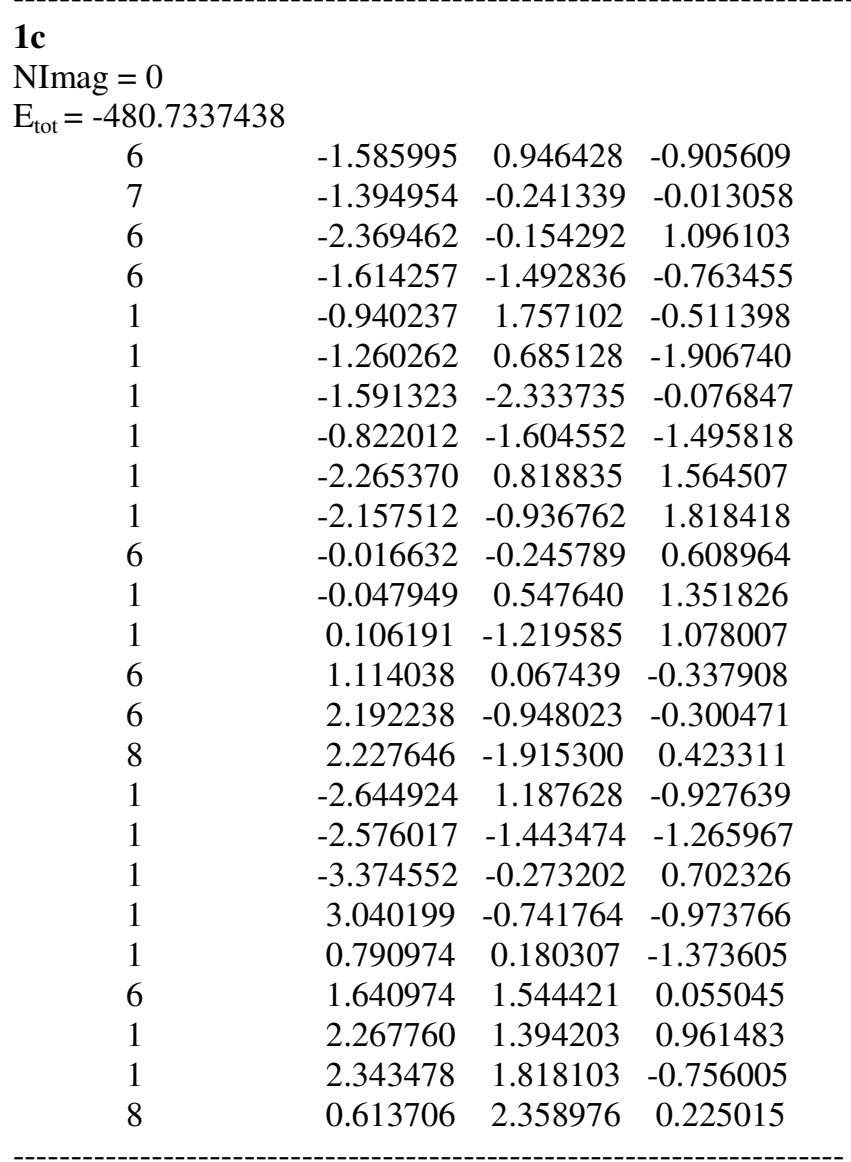

\begin{tabular}{|c|c|c|c|}
\hline \multicolumn{4}{|c|}{$\begin{array}{l}\text { TS(1c-1d) } \\
\left.\text { NImag = 1(-1460.3 } \mathrm{cm}^{-1}\right) \\
E_{\text {tot }}=-480.6857933\end{array}$} \\
\hline 7 & 1.405387 & -0.299766 & -0.000596 \\
\hline 6 & 0.091428 & -0.346302 & -0.821145 \\
\hline 6 & -1.153394 & 0.148837 & -0.177781 \\
\hline 6 & -1.291132 & 1.552087 & 0.135258 \\
\hline 6 & -2.008410 & -0.892750 & 0.559566 \\
\hline 6 & 1.146645 & -0.323139 & 1.455192 \\
\hline 6 & 2.155884 & 0.937092 & -0.335877 \\
\hline 6 & 2.222599 & -1.475022 & -0.366055 \\
\hline 1 & 0.335195 & 0.201224 & -1.728339 \\
\hline 1 & -0.061359 & -1.393906 & -1.071244 \\
\hline 1 & -1.381398 & -1.681245 & 1.023893 \\
\hline 1 & -2.056997 & -0.193120 & -0.981326 \\
\hline 1 & 0.544834 & -1.190800 & 1.699001 \\
\hline 1 & 0.612545 & 0.577648 & 1.732114 \\
\hline 1 & 1.487684 & 1.783461 & -0.203833 \\
\hline 1 & 2.471648 & 0.879230 & -1.372959 \\
\hline 1 & 2.347040 & -1.494976 & -1.444322 \\
\hline 1 & 1.713101 & -2.377286 & -0.043374 \\
\hline 8 & -2.805664 & -1.343644 & -0.459193 \\
\hline
\end{tabular}




$\begin{array}{rrrr}8 & -0.489491 & 2.436544 & -0.129852 \\ 1 & 3.194324 & -1.404935 & 0.114658 \\ 1 & 3.027359 & 1.006542 & 0.309253 \\ 1 & 2.100777 & -0.365354 & 1.973082 \\ 1 & -2.548361 & -0.413993 & 1.398106 \\ 1 & -2.244579 & 1.817848 & 0.616873\end{array}$

$1 d$

NImag $=0$

$E_{\text {tot }}=-480.7684358$

$\begin{array}{rrrr}7 & 1.463213 & -0.288366 & -0.002269 \\ 6 & 0.077546 & -0.183993 & -0.739523 \\ 6 & -1.051969 & 0.199252 & 0.100095 \\ 6 & -1.348688 & 1.566311 & 0.168430 \\ 6 & -2.024612 & -0.826124 & 0.554568 \\ 6 & 1.376259 & -1.313628 & 1.049800 \\ 6 & 1.790037 & 1.017392 & 0.609499 \\ 6 & 2.495331 & -0.654395 & -0.984083 \\ 1 & 0.278097 & 0.567023 & -1.503625 \\ 1 & -0.037157 & -1.168315 & -1.193547 \\ 1 & -1.581638 & -1.630750 & 1.145157 \\ 1 & -2.984803 & -0.867146 & -1.125890 \\ 1 & 1.134304 & -2.270564 & 0.596843 \\ 1 & 0.585706 & -1.022246 & 1.732952 \\ 1 & 1.005844 & 1.264946 & 1.314840 \\ 1 & 1.794719 & 1.779259 & -0.160918 \\ 1 & 2.536441 & 0.111311 & -1.752518 \\ 1 & 2.236606 & -1.607298 & -1.436320 \\ 8 & -2.669516 & -1.529514 & -0.507663 \\ 8 & -0.650791 & 2.496366 & -0.267967 \\ 1 & 2.325855 & -1.378514 & 1.574701 \\ 1 & 2.755380 & 0.941112 & 1.104347 \\ 1 & 3.462305 & -0.731975 & -0.493985 \\ 1 & -2.300873 & 1.814801 & 0.674255 \\ 1 & -2.774244 & -0.346800 & 1.191908\end{array}$

TS(1d-1e)

NImag $=1\left(-252.5 \mathrm{~cm}^{-1}\right)$

$\mathrm{E}_{\mathrm{tot}}=-480.7632593$

$\begin{array}{lrrr}7 & -1.626351 & -0.264512 & -0.021922 \\ 6 & 0.096775 & -0.124762 & 0.852628 \\ 6 & 1.157151 & 0.189684 & 0.011351 \\ 6 & 1.478137 & 1.571285 & -0.171440 \\ 6 & 2.043885 & -0.867084 & -0.563124 \\ 6 & -1.541945 & -1.348304 & -0.982546 \\ 6 & -1.857308 & 1.007205 & -0.688389 \\ 6 & -2.612338 & -0.527448 & 1.007932 \\ 1 & -0.248282 & 0.664090 & 1.509567 \\ 1 & 0.044048 & -1.125767 & 1.264352 \\ 1 & 1.508489 & -1.588578 & -1.178794 \\ 1 & 3.062546 & -1.079751 & 1.070632 \\ 1 & -1.319490 & -2.280213 & -0.468109 \\ 1 & -0.740536 & -1.131036 & -1.683141 \\ 1 & -1.032036 & 1.201149 & -1.367064 \\ 1 & -1.877755 & 1.807071 & 0.044723 \\ 1 & -2.602944 & 0.283448 & 1.732395 \\ 1 & -2.368618 & -1.456901 & 1.518324 \\ 8 & 2.687042 & -1.669648 & 0.414183 \\ 8 & 0.819201 & 2.528772 & 0.214530 \\ 1 & -2.477922 & -1.459772 & -1.535246 \\ 1 & -2.796335 & 0.986387 & -1.246876 \\ 1 & -3.617245 & -0.610469 & 0.587105 \\ 1 & 2.425230 & 1.761043 & -0.710786 \\ 1 & 2.789213 & -0.395567 & -1.211808\end{array}$


1e

NImag $=0$

$\mathrm{E}_{\mathrm{tot}}=-480.7776431$

$\begin{array}{lrrr}7 & -2.465441 & -0.354013 & 0.004111 \\ 6 & 0.993868 & -0.801175 & -0.070859 \\ 6 & 1.934863 & 0.140774 & -0.051280 \\ 6 & 1.560539 & 1.568227 & 0.028242 \\ 6 & 3.410727 & -0.147945 & -0.112807 \\ 6 & -3.377815 & -1.467931 & -0.011038 \\ 6 & -2.596871 & 0.451398 & -1.185142 \\ 6 & -2.629283 & 0.445984 & 1.192826 \\ 1 & -0.061034 & -0.556380 & -0.021592 \\ 1 & 1.267760 & -1.843900 & -0.148554 \\ 1 & 3.804703 & 0.130842 & -1.090226 \\ 1 & 3.494050 & -1.769267 & 0.939720 \\ 1 & -3.222626 & -2.087144 & 0.871327 \\ 1 & -3.198574 & -2.082722 & -0.891887 \\ 1 & -2.416540 & -0.161059 & -2.067756 \\ 1 & -1.860850 & 1.252129 & -1.166979 \\ 1 & -1.893892 & 1.247429 & 1.197930 \\ 1 & -2.472311 & -0.170256 & 2.077317 \\ 8 & 3.735756 & -1.496623 & 0.053045 \\ 8 & 0.434525 & 1.995478 & 0.058441 \\ 1 & -4.432917 & -1.152161 & -0.024526 \\ 1 & -3.598008 & 0.900086 & -1.280680 \\ 1 & -3.633151 & 0.893333 & 1.263762 \\ 1 & 2.407701 & 2.275858 & 0.060940 \\ 1 & 3.935365 & 0.474461 & 0.620894\end{array}$

$\mathrm{NMe}_{3}$

NImag $=\mathbf{0}$

$E_{\text {tot }}=-174.4333783$

$\begin{array}{rrrr}6 & 1.228741 & -0.614063 & 0.059876 \\ 7 & 0.000060 & -0.000075 & -0.369085 \\ 6 & -1.146206 & -0.756973 & 0.060013 \\ 6 & -0.082543 & 1.371060 & 0.060169 \\ 1 & 1.290061 & -1.629392 & -0.328473 \\ 1 & 2.077770 & -0.053577 & -0.328036 \\ 1 & -0.992406 & 1.826356 & -0.327572 \\ 1 & 0.766042 & 1.932316 & -0.327636 \\ 1 & -1.084886 & -1.772870 & -0.326841 \\ 1 & -2.056313 & -0.303271 & -0.329059 \\ 1 & 1.322332 & -0.661004 & 1.156783 \\ 1 & -0.088759 & 1.475220 & 1.157125 \\ 1 & -1.234208 & -0.813396 & 1.156960\end{array}$

Acrylaldehyde

NImag $=\mathbf{0}$

$E_{\text {tot }}=-191.8479761$

$\begin{array}{rrrr}6 & 1.457968 & -0.464952 & 0.000029 \\ 1 & 2.533688 & -0.371547 & 0.000216 \\ 1 & 1.036360 & -1.460216 & 0.000432 \\ 6 & 0.661781 & 0.599426 & -0.000188 \\ 6 & -0.807754 & 0.460314 & 0.000106 \\ 1 & 1.061037 & 1.605000 & -0.000183 \\ 8 & -1.390924 & -0.593217 & -0.000105 \\ 1 & -1.375670 & 1.403771 & 0.000693\end{array}$

Formaldehyde

NImag $=0$

$E_{\text {tot }}=-114.4602254$

$\begin{array}{rrrr}6 & -0.000001 & -0.525787 & 0.000000 \\ 8 & -0.000001 & 0.670349 & -0.000000\end{array}$

S- 22 - 


$\begin{array}{llll}0.000005 & -1.104033 & 0.934805 \\ 1 & 0.000005 & -1.104033 & -0.934805\end{array}$

S5. The CBS-4M optimized coordinates, electronic energy ( $E_{\text {tot }}$ in hartree/particle) and number of imaginary frequencies of the model system.

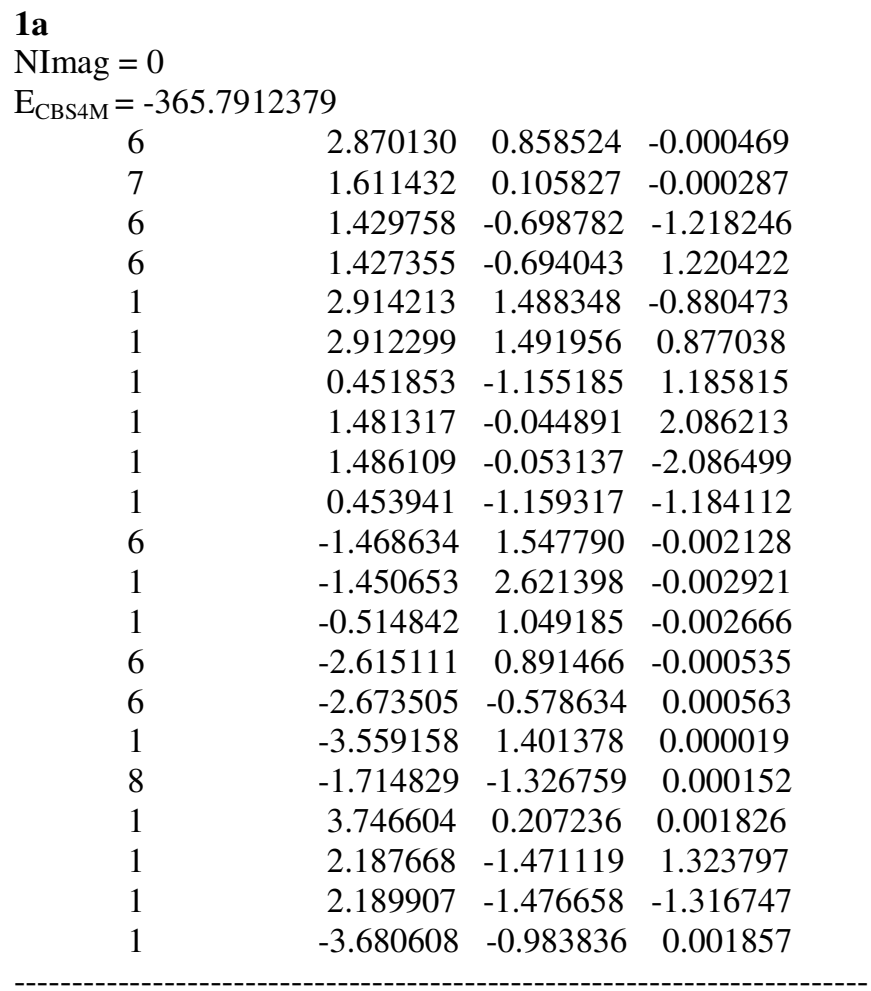

\begin{tabular}{|c|c|c|c|}
\hline $\begin{array}{l}\text { TS(1a-1b) } \\
\text { NImag = } \\
\mathrm{E}_{\mathrm{CBS} 4 \mathrm{M}}=-\end{array}$ & & & \\
\hline 6 & 1.832339 & 1.043819 & 0.704749 \\
\hline 7 & 1.118258 & -0.069357 & 0.036508 \\
\hline 6 & 1.990812 & -0.802221 & -0.910891 \\
\hline 6 & 0.506038 & -0.986680 & 1.039666 \\
\hline 1 & 2.292041 & 1.675664 & -0.043028 \\
\hline 1 & 1.106290 & 1.616614 & 1.262821 \\
\hline 1 & -0.177539 & -1.651220 & 0.538281 \\
\hline 1 & -0.068103 & -0.388646 & 1.728655 \\
\hline 1 & 2.341030 & -0.119384 & -1.673392 \\
\hline 1 & 1.417032 & -1.592484 & -1.373347 \\
\hline 6 & -0.264869 & 0.653027 & -0.91577 \\
\hline 1 & 0.335642 & 1.372312 & -1.446333 \\
\hline 1 & -0.469888 & -0.244035 & -1.47385 \\
\hline 6 & -1.333562 & 1.110892 & -0.13435 \\
\hline 6 & -2.367427 & 0.197865 & 0.154147 \\
\hline 1 & -1.414883 & 2.136538 & 0.161072 \\
\hline 8 & -2.356013 & -1.020227 & -0.09238 \\
\hline 1 & 2.596215 & 0.662534 & 1.37338 \\
\hline 1 & 1.284137 & -1.526220 & 1.568199 \\
\hline 1 & 2.842465 & -1.232384 & -0.39548 \\
\hline 1 & -3.244124 & 0.637813 & 0.63131 \\
\hline
\end{tabular}

$\mathbf{1 b}$

NImag $=0$

$\mathrm{E}_{\mathrm{CBS} 4 \mathrm{M}}=-365.772005$

$\begin{array}{rrrr}6 & 1.781427 & 1.071457 & 0.673329 \\ 7 & 1.054526 & -0.057607 & 0.024061 \\ 6 & 1.963794 & -0.851391 & -0.852808\end{array}$




$\begin{array}{lrrr}6 & 0.429003 & -0.942295 & 1.073635 \\ 1 & 2.272481 & 1.661987 & -0.086929 \\ 1 & 1.049586 & 1.673034 & 1.189193 \\ 1 & -0.344762 & -1.530710 & 0.608606 \\ 1 & -0.044410 & -0.303615 & 1.799117 \\ 1 & 2.334451 & -0.214904 & -1.643555 \\ 1 & 1.403017 & -1.670427 & -1.277075 \\ 6 & -0.156548 & 0.561843 & -0.904370 \\ 1 & 0.392156 & 1.258994 & -1.522606 \\ 1 & -0.449745 & -0.318916 & -1.456619 \\ 6 & -1.270594 & 1.107955 & -0.160410 \\ 6 & -2.316705 & 0.237893 & 0.113207 \\ 1 & -1.353367 & 2.159934 & 0.017797 \\ 8 & -2.315735 & -1.005169 & -0.076932 \\ 1 & 2.513606 & 0.683910 & 1.369219 \\ 1 & 1.205311 & -1.538154 & 1.534631 \\ 1 & 2.792001 & -1.235995 & -0.272963 \\ 1 & -3.208391 & 0.706700 & 0.532715\end{array}$

\begin{tabular}{|c|c|c|c|}
\hline \multicolumn{4}{|c|}{$\begin{array}{l}\text { TS(1b-1c) } \\
\text { NImag }=1\left(-117.2 \mathrm{~cm}^{-1}\right) \\
E_{\text {CBS4M }}=-480.1232069\end{array}$} \\
\hline 6 & -1.671336 & -1.135511 & 1.125917 \\
\hline 7 & -1.446433 & -0.149898 & 0.011405 \\
\hline 6 & -2.340406 & -0.500488 & -1.146725 \\
\hline 6 & -1.740898 & 1.251992 & 0.476098 \\
\hline 1 & -1.391393 & -2.116521 & 0.775214 \\
\hline 1 & -1.045805 & -0.865295 & 1.959060 \\
\hline 1 & -1.386144 & 1.941423 & -0.272058 \\
\hline 1 & -1.200514 & 1.426970 & 1.391328 \\
\hline 1 & -2.070538 & -1.484008 & -1.498771 \\
\hline 1 & -2.185235 & 0.224513 & -1.930513 \\
\hline 6 & 0.016782 & -0.263479 & -0.488147 \\
\hline 1 & 0.227676 & -1.318032 & -0.672942 \\
\hline 1 & 0.055242 & 0.331342 & -1.387707 \\
\hline 6 & 1.093167 & 0.172037 & 0.452487 \\
\hline 6 & 1.501180 & 1.566493 & 0.315342 \\
\hline 8 & 1.065552 & 2.357399 & -0.511334 \\
\hline 1 & -2.712483 & -1.108706 & 1.414777 \\
\hline 1 & -2.804041 & 1.351839 & 0.643777 \\
\hline 1 & -3.370365 & -0.484563 & -0.820512 \\
\hline 1 & 2.295342 & 1.872932 & 0.994029 \\
\hline 1 & 0.969781 & -0.140741 & 1.477532 \\
\hline 6 & 2.391756 & -0.949752 & -0.048241 \\
\hline 1 & 2.821635 & -0.409520 & -0.902153 \\
\hline 1 & 3.077428 & -0.830360 & 0.797897 \\
\hline 8 & 1.852318 & -2.131116 & -0.259813 \\
\hline
\end{tabular}

\begin{tabular}{|c|c|c|c|}
\hline \multicolumn{4}{|c|}{$\begin{array}{l}\text { 1c } \\
\text { NImag }=0 \\
E_{\mathrm{CBS} 4 \mathrm{M}}=-480.1286933\end{array}$} \\
\hline 7 & 1.193913 & 0.143037 & -0.025563 \\
\hline 6 & 0.095499 & -0.088542 & -1.098450 \\
\hline 6 & -1.367066 & -0.072175 & -0.684478 \\
\hline 6 & -1.898261 & 1.153569 & -0.051254 \\
\hline 6 & -1.633856 & -1.434012 & 0.210766 \\
\hline 6 & 0.731285 & -0.102119 & 1.407592 \\
\hline 6 & 1.744335 & 1.538895 & -0.140330 \\
\hline 6 & 2.268522 & -0.883927 & -0.300877 \\
\hline 1 & 0.307732 & 0.628140 & -1.877395 \\
\hline 1 & 0.246416 & -1.098160 & -1.429640 \\
\hline 1 & -1.942425 & -1.061426 & 1.198624 \\
\hline 1 & -1.914360 & -0.208453 & -1.615607 \\
\hline 1 & 0.306097 & -1.103620 & 1.370976 \\
\hline
\end{tabular}




$\begin{array}{rrc}0.020168 & 0.661179 & 1.662226 \\ 0.925177 & 2.216360 & 0.024453 \\ 2.164904 & 1.670671 & -1.126890 \\ 2.625349 & -0.755234 & -1.312611 \\ 1.790723 & -1.842160 & -0.169463 \\ -0.519573 & -2.228331 & 0.211196 \\ -1.257674 & 2.110858 & 0.352117 \\ 3.077123 & -0.739518 & 0.400250 \\ 2.513251 & 1.669339 & 0.607002 \\ 1.604470 & -0.024229 & 2.040520 \\ -2.527927 & -1.885699 & -0.228290 \\ -2.978854 & 1.141202 & 0.070461\end{array}$

\section{TS(1c-1d)}

NImag $=\left(-1712.6 \mathrm{~cm}^{-1}\right)$

$\mathrm{E}_{\mathrm{CBS} 4 \mathrm{M}}=-480.0820392$

$\begin{array}{lrrr}7 & 1.354206 & -0.332533 & 0.013027 \\ 6 & -0.006056 & -0.396235 & -0.787072 \\ 6 & -1.228443 & 0.241927 & -0.196677 \\ 6 & -1.247501 & 1.659819 & 0.052557 \\ 6 & -2.082137 & -0.766066 & 0.633388 \\ 6 & 1.131669 & -0.095249 & 1.482152 \\ 6 & 2.187099 & 0.807350 & -0.533819 \\ 6 & 2.082029 & -1.632159 & -0.182722 \\ 1 & 0.253925 & 0.026871 & -1.745805 \\ 1 & -0.262956 & -1.438367 & -0.900397 \\ 1 & -1.510290 & -1.321783 & 1.378578 \\ 1 & -2.064840 & -0.272940 & -0.995803 \\ 1 & 0.489985 & -0.866055 & 1.870172 \\ 1 & 0.671000 & 0.869158 & 1.597947 \\ 1 & 1.574090 & 1.691901 & -0.482206 \\ 1 & 2.443957 & 0.579862 & -1.557589 \\ 1 & 2.208859 & -1.801314 & -1.241349 \\ 1 & 1.492095 & -2.427704 & 0.245589 \\ 8 & -2.537641 & -1.540345 & -0.482517 \\ 8 & -0.332636 & 2.459974 & -0.144755 \\ 1 & 3.046410 & -1.576468 & 0.301598 \\ 1 & 3.084755 & 0.899712 & 0.061037 \\ 1 & 2.091961 & -0.115532 & 1.978151 \\ 1 & -2.860817 & -0.216805 & 1.168736 \\ 1 & -2.195324 & 2.023842 & 0.441482\end{array}$

\begin{tabular}{crrr} 
1d & \multicolumn{4}{c}{ NImag $=0$} & & & \\
$\mathrm{E}_{\mathrm{CBS} 4 \mathrm{M}}=-480.1623076$ & & & \\
7 & -1.443398 & -0.310903 & -0.010828 \\
6 & -0.018642 & -0.170419 & 0.731914 \\
6 & 1.093491 & 0.261626 & -0.110090 \\
6 & 1.323361 & 1.629673 & -0.145604 \\
6 & 2.081477 & -0.757768 & -0.571805 \\
6 & -1.429730 & -1.521544 & -0.888317 \\
6 & -1.671678 & 0.929600 & -0.843100 \\
6 & -2.506048 & -0.433610 & 1.034505 \\
1 & -0.243813 & 0.575841 & 1.480005 \\
1 & 0.131156 & -1.148453 & 1.166665 \\
1 & 1.685139 & -1.465217 & -1.290589 \\
1 & 2.881742 & -1.064053 & 1.220848 \\
1 & -1.283012 & -2.399922 & -0.277211 \\
1 & -0.611975 & -1.418794 & -1.581135 \\
1 & -1.035866 & 0.864029 & -1.709290 \\
1 & -1.353703 & 1.785918 & -0.270720 \\
1 & -2.513879 & 0.470414 & 1.623909 \\
1 & -2.279794 & -1.280889 & 1.665431 \\
8 & 2.547342 & -1.628071 & 0.509132
\end{tabular}




$\begin{array}{rrrr}8 & 0.559420 & 2.523732 & 0.298040 \\ 1 & -2.368439 & -1.590777 & -1.421369 \\ 1 & -2.712738 & 0.965600 & -1.133082 \\ 1 & -3.467919 & -0.572641 & 0.560694 \\ 1 & 2.266394 & 1.924623 & -0.613959 \\ 1 & 2.923009 & -0.259996 & -1.046791\end{array}$

\begin{tabular}{|c|c|c|c|}
\hline \multicolumn{4}{|c|}{$\begin{array}{l}\text { TS(1d-1e) } \\
\left.\text { NImag = 1(-320.5 } \mathrm{cm}^{-1}\right) \\
E_{C B S 4 M}=-480.1590937\end{array}$} \\
\hline 7 & 1.548718 & -0.285206 & 0.035215 \\
\hline 6 & -0.131006 & -0.110943 & -0.838789 \\
\hline 6 & -1.192865 & 0.262535 & -0.023186 \\
\hline 6 & -1.431932 & 1.650308 & 0.133229 \\
\hline 6 & -2.102401 & -0.780798 & 0.558913 \\
\hline 6 & 1.598786 & -1.600990 & 0.714022 \\
\hline 6 & 1.619896 & 0.834770 & 1.009147 \\
\hline 6 & 2.595637 & -0.169904 & -1.005624 \\
\hline 1 & 0.240010 & 0.649865 & -1.499015 \\
\hline 1 & -0.130407 & -1.111507 & -1.229189 \\
\hline 1 & -1.656132 & -1.335417 & 1.374467 \\
\hline 1 & -2.836656 & -1.393633 & -1.181084 \\
\hline 1 & 1.508998 & -2.388238 & -0.021660 \\
\hline 1 & 0.771710 & -1.666515 & 1.403699 \\
\hline 1 & 0.854281 & 0.683952 & 1.754007 \\
\hline 1 & 1.403593 & 1.757465 & 0.496450 \\
\hline 1 & 2.520513 & 0.800697 & -1.474774 \\
\hline 1 & 2.444313 & -0.940445 & -1.750141 \\
\hline 8 & -2.443996 & -1.817256 & -0.404952 \\
\hline 8 & -0.685247 & 2.565210 & -0.237698 \\
\hline 1 & 2.532661 & -1.720271 & 1.254905 \\
\hline 1 & 2.595979 & 0.860385 & 1.482901 \\
\hline 1 & 3.585052 & -0.280724 & -0.573374 \\
\hline 1 & -2.377242 & 1.895347 & 0.622372 \\
\hline 1 & -3.000439 & -0.308021 & 0.948843 \\
\hline
\end{tabular}

\section{1e}

$\mathrm{NImag}=0$

$\mathrm{E}_{\mathrm{CBS} 4 \mathrm{M}}=-480.1754511$

$\begin{array}{lrrr}7 & -2.409823 & -0.367840 & -0.004455 \\ 6 & 0.968606 & -0.802425 & -0.119005 \\ 6 & 1.873088 & 0.157354 & -0.066903 \\ 6 & 1.474813 & 1.572553 & 0.037566 \\ 6 & 3.360656 & -0.122939 & -0.139167 \\ 6 & -3.374210 & -1.471667 & -0.049848 \\ 6 & -2.498137 & 0.513810 & -1.178238 \\ 6 & -2.465834 & 0.385857 & 1.256888 \\ 1 & -0.088044 & -0.609374 & -0.075370 \\ 1 & 1.285623 & -1.822416 & -0.213898 \\ 1 & 3.737254 & 0.105684 & -1.126702 \\ 1 & 3.486360 & -1.751755 & 0.992242 \\ 1 & -3.208594 & -2.131128 & 0.793201 \\ 1 & -3.231472 & -2.038611 & -0.961486 \\ 1 & -2.362406 & -0.073123 & -2.078692 \\ 1 & -1.710765 & 1.249873 & -1.116309 \\ 1 & -1.678631 & 1.124713 & 1.251821 \\ 1 & -2.308723 & -0.292172 & 2.087324 \\ 8 & 3.664882 & -1.511831 & 0.072471 \\ 8 & 0.336195 & 1.993872 & 0.083480 \\ 1 & -4.409059 & -1.124406 & -0.017956 \\ 1 & -3.461674 & 1.024101 & -1.240639 \\ 1 & -3.426080 & 0.886647 & 1.397739 \\ 1 & 2.310218 & 2.269010 & 0.074087 \\ 1 & 3.892255 & 0.506252 & 0.570466\end{array}$




\begin{tabular}{|c|c|c|c|}
\hline \multicolumn{4}{|c|}{$\begin{array}{l}\mathbf{N M e}_{3} \\
\text { NImag }=0 \\
E_{\text {CBS4M }}=-174.1585786\end{array}$} \\
\hline 6 & -1.023021 & -0.970125 & 0.057149 \\
\hline 7 & -0.000001 & -0.000007 & -0.337261 \\
\hline 6 & -0.328644 & 1.371025 & 0.057101 \\
\hline 6 & 1.351666 & -0.400897 & 0.057132 \\
\hline 1 & -1.980895 & -0.668818 & -0.348632 \\
\hline 1 & -0.772914 & -1.942643 & -0.348584 \\
\hline 1 & 2.068832 & 0.301949 & -0.348633 \\
\hline 1 & 1.569653 & -1.381104 & -0.348628 \\
\hline 1 & -1.295918 & 1.640675 & -0.348652 \\
\hline 1 & 0.411239 & 2.049900 & -0.348692 \\
\hline 1 & -1.122214 & -1.064151 & 1.141477 \\
\hline 1 & 1.482712 & -0.439759 & 1.141458 \\
\hline 1 & -0.360490 & 1.503978 & 1.141425 \\
\hline
\end{tabular}

Acrylaldehyde
NImag $=0$
$\begin{array}{rrrr}\mathrm{E}_{\mathrm{CBS} 4 \mathrm{M}}= & -191.6285365 & & \\ 6 & -1.439206 & -0.471541 & 0.000005 \\ 1 & -2.509591 & -0.413043 & 0.000011 \\ 1 & -0.998399 & -1.450218 & -0.000044 \\ 6 & -0.672020 & 0.600352 & -0.000001 \\ 6 & 0.800311 & 0.469110 & -0.000022 \\ 1 & -1.075552 & 1.593709 & 0.000027 \\ 8 & 1.388514 & -0.590907 & 0.000011 \\ 1 & 1.340920 & 1.409286 & 0.000023\end{array}$

\section{Formaldehyde}

NImag $=0$

$\mathrm{E}_{\mathrm{CBS} 4 \mathrm{M}}=-114.3600544$

$\begin{array}{rrrr}6 & -0.000000 & -0.530858 & 0.000000 \\ 8 & -0.000000 & 0.676311 & -0.000000 \\ 1 & 0.000001 & -1.112672 & 0.913356 \\ 1 & 0.000001 & -1.112672 & -0.913356\end{array}$

S6. The MP2/6-31+G* optimized coordinates and ground state electronic energy $\left(\mathrm{E}_{\text {tot }}\right.$ in hartree/particle) of the model system.

$\begin{array}{crrr}\mathbf{1 a} & & & \\ \text { NImag }=0 & & & \\ \mathrm{E}_{\text {tot }}=-365.1705829 & & & \\ 6 & 2.850274 & 0.891731 & -0.042784 \\ 7 & 1.609337 & 0.128568 & -0.017164 \\ 6 & 1.556197 & -0.808594 & -1.134714 \\ 6 & 1.466668 & -0.581402 & 1.249789 \\ 1 & 2.922468 & 1.441535 & -0.985917 \\ 1 & 2.854756 & 1.610352 & 0.782210 \\ 1 & 0.519930 & -1.127137 & 1.250771 \\ 1 & 1.462249 & 0.140620 & 2.072418 \\ 1 & 1.624392 & -0.255021 & -2.076266 \\ 1 & 0.605551 & -1.346687 & -1.106905 \\ 6 & -1.524469 & 1.553659 & -0.083945 \\ 1 & -1.529228 & 2.640120 & -0.112597 \\ 1 & -0.556625 & 1.052092 & -0.106508 \\ 6 & -2.683642 & 0.870572 & -0.021620 \\ 6 & -2.725223 & -0.606861 & 0.019133 \\ 1 & -3.643329 & 1.384812 & 0.001074 \\ 8 & -1.731376 & -1.336736 & 0.006335 \\ 1 & 3.743945 & 0.244671 & 0.053187 \\ 1 & 2.292071 & -1.299435 & 1.420313\end{array}$




\section{TS(1a-1b)}

NImag $=1\left(-239.8 \mathrm{~cm}^{-1}\right)$

$\mathrm{E}_{\mathrm{tot}}=-365.1560673$

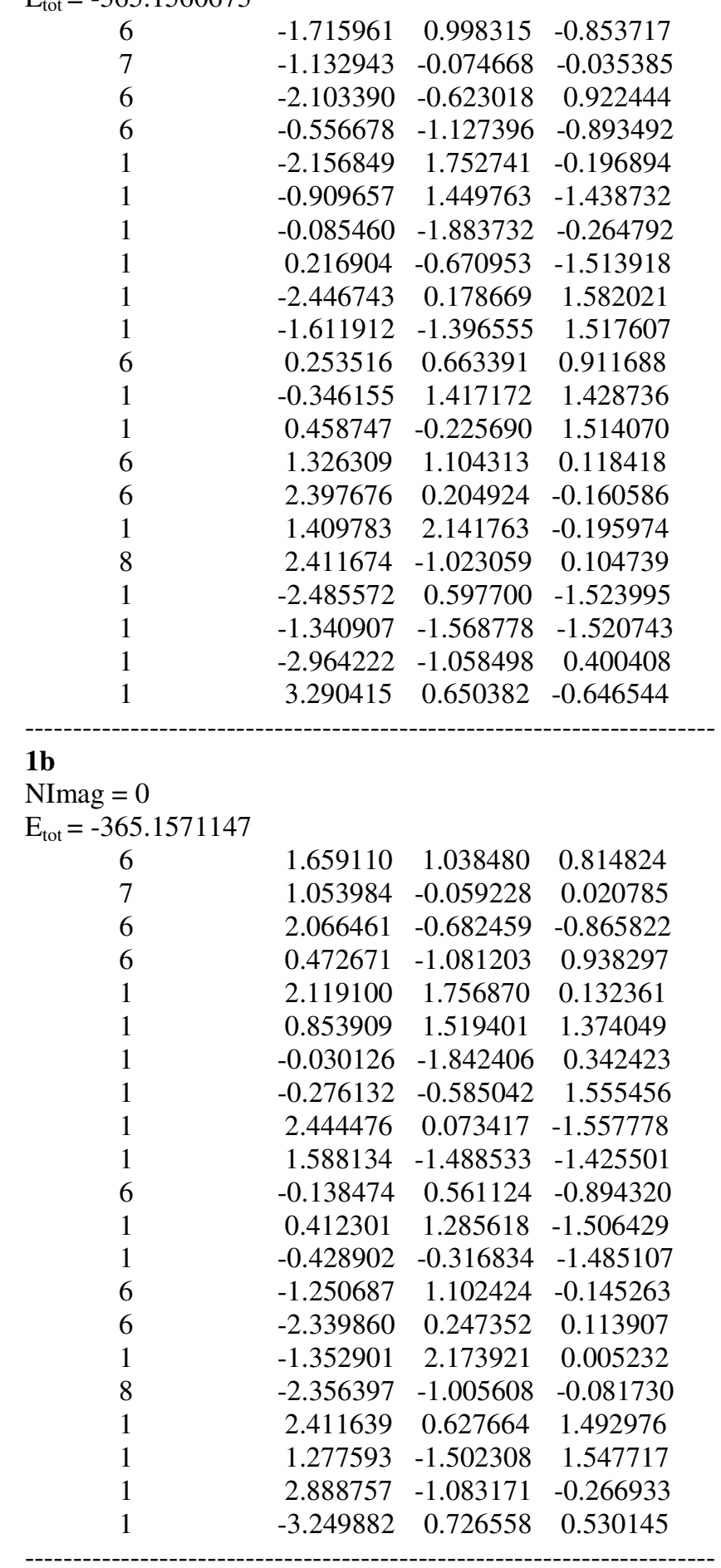

TS(1b-1c)

NImag $=1\left(-215.5 \mathrm{~cm}^{-1}\right)$

$\mathrm{E}_{\mathrm{tot}}=-479.3302347$

$\begin{array}{lrrr}6 & 1.617826 & 1.039493 & 0.959957 \\ 7 & 1.445990 & -0.106334 & 0.003686 \\ 6 & 2.411593 & 0.061924 & -1.124050 \\ 6 & 1.699618 & -1.404901 & 0.691621 \\ 1 & 1.113713 & 1.914376 & 0.537089 \\ 1 & 1.144925 & 0.783379 & 1.906714 \\ 1 & 1.634555 & -2.207531 & -0.045193 \\ 1 & 0.930114 & -1.544981 & 1.450667\end{array}$




$\begin{array}{rrr}2.245178 & 1.042265 & -1.573492 \\ 2.227260 & -0.722205 & -1.860216 \\ 0.030678 & -0.076473 & -0.586813 \\ 0.007868 & 0.877435 & -1.133232 \\ -0.038270 & -0.932173 & -1.267270 \\ -1.065896 & -0.123351 & 0.414772 \\ -1.898367 & -1.301745 & 0.384245 \\ -1.933666 & -2.145262 & -0.537618 \\ 2.686881 & 1.205058 & 1.112217 \\ 2.692132 & -1.378625 & 1.148002 \\ 3.431454 & -0.010873 & -0.739815 \\ -2.620672 & -1.399064 & 1.221600 \\ -0.862415 & 0.248197 & 1.417309 \\ -2.001588 & 1.495104 & -0.078223 \\ -2.513189 & 1.114476 & -0.986011 \\ -2.688635 & 1.538259 & 0.786569 \\ -1.100835 & 2.404768 & -0.208607\end{array}$

$1 \mathrm{c}$

NImag $=0$

$\mathrm{E}_{\mathrm{tot}}=-479.3373723$

$\begin{array}{lrrr}7 & -1.195831 & -0.141555 & -0.017329 \\ 6 & -0.088902 & 0.008781 & -1.083146 \\ 6 & 1.362106 & 0.098793 & -0.653013 \\ 6 & 1.945554 & -1.119985 & -0.054633 \\ 6 & 1.593705 & 1.429733 & 0.259604 \\ 6 & -0.751392 & 0.023648 & 1.412836 \\ 6 & -1.833529 & -1.489167 & -0.159636 \\ 6 & -2.212742 & 0.933036 & -0.282116 \\ 1 & -0.236095 & -0.828070 & -1.772567 \\ 1 & -0.298676 & 0.955710 & -1.579281 \\ 1 & 1.777563 & 1.057208 & 1.299750 \\ 1 & 1.906723 & 0.299344 & -1.589291 \\ 1 & -0.342967 & 1.036382 & 1.485976 \\ 1 & -0.025486 & -0.752425 & 1.642683 \\ 1 & -1.065654 & -2.244193 & 0.012343 \\ 1 & -2.244911 & -1.579642 & -1.166776 \\ 1 & -2.585846 & 0.813974 & -1.301412 \\ 1 & -1.688723 & 1.884740 & -0.157763 \\ 8 & 0.540396 & 2.262394 & 0.132957 \\ 8 & 1.307869 & -2.119870 & 0.307548 \\ 1 & -3.031757 & 0.820906 & 0.431733 \\ 1 & -2.635154 & -1.575432 & 0.576426 \\ 1 & -1.639842 & -0.101700 & 2.036578 \\ 1 & 2.567074 & 1.845652 & -0.075269 \\ 1 & 3.039638 & -1.090791 & 0.114750\end{array}$

\section{TS(1c-1d)}

NImag $=1\left(-1488.8 \mathrm{~cm}^{-1}\right)$

$\mathrm{E}_{\mathrm{tot}}=-479.2859812$

$\begin{array}{lrrr}7 & 1.378553 & -0.329778 & -0.004253 \\ 6 & 0.057354 & -0.318443 & -0.855059 \\ 6 & -1.175785 & 0.191824 & -0.196701 \\ 6 & -1.234761 & 1.602700 & 0.134131 \\ 6 & -2.015885 & -0.838693 & 0.576667 \\ 6 & 1.131286 & -0.090243 & 1.453091 \\ 6 & 2.301166 & 0.739964 & -0.508403 \\ 6 & 2.031330 & -1.664337 & -0.168510 \\ 1 & 0.319772 & 0.262440 & -1.743581 \\ 1 & -0.121983 & -1.359220 & -1.142217 \\ 1 & -1.379155 & -1.624966 & 1.044391 \\ 1 & -2.100453 & -0.135999 & -0.996432 \\ 1 & 0.406039 & -0.816766 & 1.815440 \\ 1 & 0.750317 & 0.921482 & 1.579113\end{array}$




\begin{tabular}{|c|c|c|c|}
\hline 1 & 1.763549 & 1.688269 & -0.463323 \\
\hline 1 & 2.572506 & 0.504494 & -1.538969 \\
\hline 1 & 2.180059 & -1.851341 & -1.233341 \\
\hline 1 & 1.373414 & -2.426300 & 0.252885 \\
\hline 8 & -2.840326 & -1.328313 & -0.445410 \\
\hline 8 & -0.350720 & 2.443326 & -0.133746 \\
\hline 1 & 2.992733 & -1.661246 & 0.350240 \\
\hline 1 & 3.195953 & 0.756588 & 0.118359 \\
\hline 1 & 2.083233 & -0.204196 & 1.977305 \\
\hline 1 & -2.555743 & -0.343851 & 1.412857 \\
\hline 1 & -2.169977 & 1.942312 & 0.619002 \\
\hline \multicolumn{4}{|l|}{ 1d } \\
\hline \multicolumn{4}{|c|}{ NImag $=0$} \\
\hline \multicolumn{4}{|c|}{$E_{\text {tot }}=-479.3675938$} \\
\hline 7 & 1.469178 & -0.289325 & -0.005514 \\
\hline 6 & 0.066272 & -0.193746 & -0.734391 \\
\hline 6 & -1.044309 & 0.187896 & 0.136758 \\
\hline 6 & -1.356058 & 1.564381 & 0.184165 \\
\hline 6 & -2.027974 & -0.838071 & 0.571433 \\
\hline 6 & 1.397393 & -1.335212 & 1.050442 \\
\hline 6 & 1.792701 & 1.028672 & 0.621082 \\
\hline 6 & 2.510046 & -0.642798 & -1.007565 \\
\hline 1 & 0.253175 & 0.563505 & -1.507159 \\
\hline 1 & -0.053823 & -1.187345 & -1.184523 \\
\hline 1 & -1.590353 & -1.681609 & 1.119429 \\
\hline 1 & -3.025425 & -0.798076 & -1.121019 \\
\hline 1 & 1.145363 & -2.290014 & 0.583967 \\
\hline 1 & 0.613861 & -1.043000 & 1.750509 \\
\hline 1 & 1.002198 & 1.262477 & 1.333351 \\
\hline 1 & 1.790671 & 1.794679 & -0.153547 \\
\hline 1 & 2.534885 & 0.137381 & -1.770370 \\
\hline 1 & 2.249553 & -1.599800 & -1.464129 \\
\hline 8 & -2.699575 & -1.510019 & -0.538286 \\
\hline 8 & -0.645540 & 2.504471 & -0.281887 \\
\hline 1 & 2.363173 & -1.401984 & 1.557789 \\
\hline 1 & 2.765629 & 0.947404 & 1.113610 \\
\hline 1 & 3.483064 & -0.714876 & -0.515421 \\
\hline 1 & -2.308347 & 1.825439 & 0.696227 \\
\hline 1 & -2.775372 & -0.371244 & 1.229726 \\
\hline
\end{tabular}

\section{TS(1d-1e)}

NImag $=1\left(-209.9 \mathrm{~cm}^{-1}\right)$

$\mathrm{E}_{\mathrm{tot}}=-479.3619588$

$\begin{array}{lrrr}7 & -1.637168 & -0.271436 & -0.021007 \\ 6 & 0.118565 & -0.133554 & 0.867117 \\ 6 & 1.169108 & 0.189777 & 0.013096 \\ 6 & 1.481578 & 1.585919 & -0.173475 \\ 6 & 2.057281 & -0.861718 & -0.577973 \\ 6 & -1.637337 & -1.498191 & -0.824402 \\ 6 & -1.761722 & 0.917257 & -0.872468 \\ 6 & -2.656829 & -0.310047 & 1.029943 \\ 1 & -0.228528 & 0.659199 & 1.531054 \\ 1 & 0.071108 & -1.143950 & 1.276584 \\ 1 & 1.516035 & -1.600452 & -1.177356 \\ 1 & 3.090482 & -1.052638 & 1.080379 \\ 1 & -1.477304 & -2.360731 & -0.171890 \\ 1 & -0.822184 & -1.438761 & -1.549275 \\ 1 & -0.898670 & 0.949656 & -1.543010 \\ 1 & -1.740398 & 1.811928 & -0.248392 \\ 1 & -2.600650 & 0.611774 & 1.614483 \\ 1 & -2.464077 & -1.164699 & 1.685236 \\ 8 & 2.726338 & -1.670783 & 0.419458 \\ 8 & 0.785949 & 2.549404 & 0.209828\end{array}$


$1 \mathrm{e}$

NImag $=0$

$\mathrm{E}_{\mathrm{tot}}=-479.3704583$

$\begin{array}{lrrr}7 & 2.037048 & -0.326397 & -0.042280 \\ 6 & -0.771227 & -0.142121 & -1.184726 \\ 6 & -1.604307 & 0.231420 & -0.193532 \\ 6 & -1.640911 & 1.649540 & 0.235360 \\ 6 & -2.517152 & -0.726408 & 0.535763 \\ 6 & 3.030047 & -1.363767 & -0.286357 \\ 6 & 1.568202 & -0.375623 & 1.335006 \\ 6 & 2.575310 & 0.993388 & -0.351976 \\ 1 & -0.143726 & 0.593955 & -1.675521 \\ 1 & -0.706760 & -1.174285 & -1.512728 \\ 1 & -2.073669 & -1.032886 & 1.489005 \\ 1 & -3.178766 & -1.733501 & -1.011370 \\ 1 & 3.339320 & -1.332652 & -1.335261 \\ 1 & 2.591651 & -2.344876 & -0.080526 \\ 1 & 1.115837 & -1.352528 & 1.532982 \\ 1 & 0.811610 & 0.399259 & 1.486586 \\ 1 & 1.799218 & 1.747721 & -0.196290 \\ 1 & 2.887348 & 1.021372 & -1.400561 \\ 8 & -2.744891 & -1.948429 & -0.165432 \\ 8 & -0.797854 & 2.496756 & -0.068466 \\ 1 & 3.931157 & -1.243595 & 0.346611 \\ 1 & 2.385421 & -0.212471 & 2.064646 \\ 1 & 3.450627 & 1.247285 & 0.277238 \\ 1 & -2.495925 & 1.943904 & 0.878029 \\ 1 & -3.470501 & -0.227103 & 0.767073\end{array}$

$\mathrm{NMe}_{3}$

NImag $=0$

$E_{\text {tot }}=-173.839502$

$\begin{array}{lrrr}6 & -1.019945 & -0.934888 & 0.064031 \\ 7 & 0.000022 & -0.000023 & -0.391209 \\ 6 & -0.299749 & 1.350700 & 0.064037 \\ 6 & 1.319646 & -0.415765 & 0.064009 \\ 1 & -1.995982 & -0.624155 & -0.319226 \\ 1 & -0.796182 & -1.933827 & -0.320853 \\ 1 & 2.072967 & 0.277375 & -0.320895 \\ 1 & 1.538522 & -1.416464 & -0.319100 \\ 1 & -1.276739 & 1.656360 & -0.320858 \\ 1 & 0.457411 & 2.040518 & -0.319288 \\ 1 & -1.079146 & -0.990453 & 1.168736 \\ 1 & 1.397451 & -0.439209 & 1.168745 \\ 1 & -0.318170 & 1.429731 & 1.168738\end{array}$

\section{Acrylaldehyde}

NImag $=0$

$E_{\text {tot }}=-191.3228916$

$\begin{array}{lrrr}6 & -1.468664 & -0.473982 & 0.000140 \\ 1 & -2.549866 & -0.381184 & -0.000381 \\ 1 & -1.041136 & -1.472593 & 0.000728 \\ 6 & -0.667819 & 0.606330 & -0.000085 \\ 6 & 0.808718 & 0.473995 & 0.000243 \\ 1 & -1.076103 & 1.615190 & -0.001018 \\ 8 & 1.406354 & -0.602429 & -0.000258 \\ 1 & 1.382861 & 1.419967 & 0.000946\end{array}$

S- 31 - 
Formaldehyde

NImag $=0$

$\mathrm{E}_{\mathrm{tot}}=-114.1770785$

$\begin{array}{lrrr}6 & -0.000001 & -0.539810 & 0.000000 \\ 8 & -0.000001 & 0.684673 & 0.000000 \\ 1 & 0.000005 & -1.119261 & 0.937927 \\ 1 & 0.000005 & -1.119261 & -0.937927\end{array}$

S7. The mPW1K/6-31+G* optimized coordinates and ground state electronic energy ( $\mathrm{E}_{\mathrm{tot}}$ in hartree/particle) of the model systems with water molecules.

TS(1b-1c) $)_{\mathbf{2}}$

NImag $=1(-208.30)$

$E_{\text {tot }}=-633.5616617$

\begin{tabular}{|c|c|c|c|}
\hline 6 & -0.756818 & 1.982386 & 1.035337 \\
\hline 7 & 0.316952 & 1.465213 & 0.156477 \\
\hline 6 & 0.424931 & 2.322744 & -1.043015 \\
\hline 6 & 1.608030 & 1.478453 & 0.878549 \\
\hline 1 & -1.721753 & 1.846284 & 0.551672 \\
\hline 1 & -0.742515 & 1.425144 & 1.964917 \\
\hline 1 & 2.393629 & 1.146779 & 0.206529 \\
\hline 1 & 1.536266 & 0.801338 & 1.722065 \\
\hline 1 & -0.531227 & 2.324902 & -1.556471 \\
\hline 1 & 1.197575 & 1.916649 & -1.688237 \\
\hline 6 & -0.027662 & 0.059442 & -0.325340 \\
\hline 1 & -0.942427 & 0.177478 & -0.906400 \\
\hline 1 & 0.780128 & -0.216829 & -0.996768 \\
\hline 6 & -0.221221 & -0.963864 & 0.720957 \\
\hline 6 & 0.855233 & -1.798060 & 1.111171 \\
\hline 8 & 1.943092 & -1.931525 & 0.541756 \\
\hline 1 & -0.561874 & 3.031979 & 1.238191 \\
\hline 1 & 1.799710 & 2.489843 & 1.227385 \\
\hline 1 & 0.684904 & 3.332990 & -0.740004 \\
\hline 1 & 0.649618 & -2.450255 & 1.974417 \\
\hline 1 & -0.969226 & -0.771469 & 1.479139 \\
\hline 6 & -1.450633 & -2.225979 & -0.316069 \\
\hline 1 & -0.655136 & -2.753834 & -0.856148 \\
\hline 1 & -1.792907 & -2.758440 & 0.579498 \\
\hline 8 & -2.254786 & -1.516866 & -0.965241 \\
\hline 1 & 2.765432 & -0.961304 & -0.676382 \\
\hline 8 & 3.100165 & -0.268399 & -1.280557 \\
\hline 1 & 3.810348 & -0.665008 & -1.783822 \\
\hline 1 & -3.109620 & -0.161148 & -0.392898 \\
\hline 8 & -3.421197 & 0.726234 & -0.101753 \\
\hline 1 & -4.338947 & 0.782132 & -0.365210 \\
\hline
\end{tabular}

TS(1c-1d) $)_{2 \mathrm{~W}}$

NImag = $1(-209.60)$

$\mathrm{E}_{\mathrm{tot}}=-633.5502618$

$\begin{array}{lrrr}7 & 0.699536 & 1.281793 & -0.046134 \\ 6 & 0.006725 & 0.131582 & 0.665537 \\ 6 & -0.808285 & -0.851715 & -0.138471 \\ 6 & -0.108301 & -2.032024 & -0.627937 \\ 6 & -1.909652 & -0.328604 & -1.075632 \\ 6 & -0.270987 & 2.378335 & -0.299258 \\ 6 & 1.328906 & 0.847241 & -1.312808 \\ 6 & 1.765174 & 1.787528 & 0.853871 \\ 1 & 0.795861 & -0.406483 & 1.181985 \\ 1 & -0.643838 & 0.588365 & 1.407005 \\ 1 & -1.502641 & 0.150792 & -1.972965 \\ 1 & -1.512534 & -1.206121 & 0.738413 \\ 1 & -0.530590 & 2.825871 & 0.654714\end{array}$




$\begin{array}{rrr}-1.181881 & 1.976808 & -0.731776 \\ 0.558917 & 0.516781 & -1.998376 \\ 2.024337 & 0.043357 & -1.102533 \\ 2.522413 & 1.016857 & 0.966187 \\ 1.319715 & 2.020742 & 1.815924 \\ -2.727502 & 0.582644 & -0.435252 \\ 1.048144 & -2.350595 & -0.409329 \\ 2.192700 & 2.686904 & 0.419099 \\ 1.860710 & 1.692756 & -1.739959 \\ 0.199065 & 3.118929 & -0.940087 \\ -2.468601 & -1.203320 & -1.432390 \\ -0.732264 & -2.723262 & -1.214906 \\ -2.590364 & -0.605540 & 1.740430 \\ -3.286098 & -1.158981 & 2.094273 \\ -2.892502 & 0.181322 & 0.505362 \\ 2.540201 & -1.655820 & 0.329024 \\ 3.248550 & -1.054879 & 0.614377 \\ 3.988180 & -1.605140 & 0.870320\end{array}$

S- 33 - 